\title{
Self-Force with a Stochastic Component from Radiation Reaction of a Scalar Charge Moving in Curved Spacetime
}

\author{
Chad R. Galley and B. L. Hu \\ Department of Physics, University of Maryland, College Park, Maryland, 20742
}

(Dated: May 16, 2005)

\begin{abstract}
We give a quantum field theoretical derivation of the scalar Abraham-Lorentz-Dirac (ALD) equation and the self-force for a scalar charged particle interacting with a quantum scalar field in curved spacetime. We regularize the causal Green's function using a quasi-local expansion in the spirit of effective field theory and obtain a regular expression for the self-force. The scalar ALD equation obtained in this way for the classical motion of the particle checks with the equation obtained by Quinn earlier [1]. We further derive a scalar ALD-Langevin equation with a classical stochastic force accounting for the effect of quantum fluctuations in the field, which causes small fluctuations on the particle trajectory. This equation will be useful for the study of stochastic motion of charges under the influence of both quantum and classical noise sources, derived either self-consistently (as done here) or put in by hand (with warnings). We show the possibility of secular effects from such stochastic influences on the trajectory that may impact on the present calculations of gravitational waveform templates.
\end{abstract}

\section{INTRODUCTION}

Interest in the problem of radiation reaction from particle motion in a curved spacetime has seen a rapid increase in recent years. The back-reaction of emitted radiation on the particle, known as the self-force, changes the particle trajectories (e.g., near a black hole) from a simple geodesic motion. The determination of the self force is essential to precision calculations of particle trajectories and the determination of waveforms from prospective astrophysical sources. Electromagnetic radiation reaction in a curved spacetime was first studied by DeWitt and Brehm [2]. The gravitational radiation reaction equation was first obtained by Mino, Sasaki and Tanaka [3] and Quinn and Wald [4] and others, notably Detweiler and Whiting [5]. The equation of motion governing a scalar charge with radiation reaction was first obtained by Quinn [1]. For an excellent review, see [6].

Parallel to this there has been detailed work devoted to particles and detectors (e.g., atoms) moving in a quantum field and in design studies of possible detection of Unruh radiation (see, e.g., 7, , 8, 9] and references therein). The introduction of worldline path integral methods (see [10] and references therein) enable one to obtain equations of motion for the charges and the field self-consistently. The introduction of open system concepts and the influence functional method enables one to derive stochastic equations with a noise source derived $a b$ initio and in a self consistent manner (See 7, 11, 12 for accelerating detectors, 10, 13, 14] for moving charges.)

In this paper, we use the worldline influence functional method to study a particle with a scalar charge moving in its own quantum scalar field in a curved spacetime. This is a generalization of results obtained in [10] to curved spacetime. We are interested in the radiation it emits and its backreaction (radiation reaction) on the trajectory of the particle and derive the equations of motion for the quantum average (expectation value) of the particle's position. For those particle trajectory histories which become sufficiently decohered, the expectation value behaves classically. The scalar ALD equation we derive in this limit checks with the result of Quinn [1]. We then include in our consideration the effect of fluctuations in the quantum field. We show how it behaves like a classical stochastic force and derive a scalar ALD-Langevin equation for the particle dynamics with a stochastic component, thus capturing the induced small fluctuations on the particle trajectory. This equation will be useful for the study of stochastic motion of charges under the influence of both quantum or classical noise sources, derived either self-consistently (as done here) or put in by hand (with warnings). For astrophysical sources with some stochastic component this effect may need to be included in more accurate calculations of waveform templates.

In Sec. 2 we describe the worldline influence functional method and how to obtain the semiclassical and stochastic particle dynamics. In Sec. 3 we discuss how to regularize the causal Green function in the spirit of effective field theory using a quasi-local expansion. In Sec. 4 we derive the scalar ALD equation. In Sec. 5 we derive the stochastic scalar ALD or the scalar ALD-Langevin equation. We discuss the non-Markovian nature of the noise induced effects and the possibility of secular effects from such stochastic influences on the trajectory. In Sec. 6 we discuss an array of issues pertinent to the present problem and approach. In Sec. 7 we summarize our findings and Appendices A-D provide further details in the derivation of certain results given in the text.

\section{RELATIVISTIC PARTICLE-FIELD DYNAMICS}

The open quantum system paradigm starts by considering a system, or universe, which is partitioned (according to natural physical arguments or some large scale separation) into two smaller systems. One subsystem, called the system, is assumed to be the one of interest and the 
other, called the environment, contains many more degrees of freedom. An accurate description of the behavior of the system variables requires knowing the influence from the environment due to their mutual interactions. A less than accurate description of the overall influence of the environment can be obtained by introducing some coarse-graining over the environmental variables.

\section{A. Worldline Influence Functional}

In a coordinate system, assume at some initial time $t_{i}$ the quantum statistical state of the combined system $\mathrm{S}$ (particle in position $z_{i}$ ) and environment $\mathrm{E}$ (quantum field $\varphi_{i}$ ) is described by a density matrix $\rho\left(z_{i}, \varphi_{i} ; z_{i}^{\prime}, \varphi_{i}^{\prime} ; t_{i}\right)$. In practice, specifying such a state is non-trivial since one requires a time-like Killing vector to define positive frequency modes and hence a Hilbert space of states. If the spacetime admits an asymptotically flat region or is conformally flat then the initial state can be constructed. But a general spacetime may not admit a time-like Killing vector. Regardless, we will sidestep these issues by working at a formal level. At some $t_{f}>t_{i}$ the density matrix is evolved to

$$
\begin{aligned}
& \rho\left(z_{f}, \phi_{f} ; z_{f}^{\prime}, \phi_{f}^{\prime} ; t_{f}\right) \\
& =\int d z_{i} d \phi_{i} \int d z_{i}^{\prime} d \phi_{i}^{\prime} K\left(z_{f}, \phi_{f}, t_{f} ; z_{i}, \phi_{i}, t_{i}\right) \\
& \quad \times \rho\left(z_{i}, \phi_{i} ; z_{i}^{\prime}, \phi_{i}^{\prime} ; t_{i}\right) K^{*}\left(z_{f}^{\prime}, \phi_{f}^{\prime}, t_{f} ; z_{i}^{\prime}, \phi_{i}^{\prime}, t_{i}\right)
\end{aligned}
$$

where $K$ is the amplitude of the time-evolution operator $\hat{U}\left(t_{f}, t_{i}\right)=\exp \left\{-\frac{i}{\hbar} \int_{t_{i}}^{t_{f}} d t \hat{H}_{S+E}[z, \phi]\right\}$ for the system plus environment and has a path integral representation given by

$$
K\left(z_{f}, \phi_{f}, t_{f} ; z_{i}, \phi_{i}, t_{i}\right)=\int_{z_{i}, \phi_{i}}^{z_{f}, \phi_{f}} \mathcal{D} z \mathcal{D} \phi e^{\frac{i}{\hbar} S_{S+E}[z, \phi]}
$$

The action describing the system plus environment can be written as the actions for the system $S_{S}[z]$ and environment $S_{E}[\phi]$ along with an interaction action $S_{i n t}[z, \phi]$ between them

$$
S_{S+E}[z, \phi]=S_{S}[z]+S_{E}[\phi]+S_{\text {int }}[z, \phi]
$$

with

$$
\begin{aligned}
S_{S}[z] & =-m_{0} \int d \tau \sqrt{-g_{\mu \nu} u^{\mu} u^{\nu}} \\
S_{E}[\phi] & =\frac{1}{2} \int d^{4} x \sqrt{-g}\left(g^{\mu \nu} \partial_{\mu} \phi \partial_{\nu} \phi-\xi_{R} R \phi^{2}\right) \\
S_{\text {int }}[z, \phi] & =\int d^{4} x j(x ; z] \phi(x) \\
& =-\frac{e}{4 \pi} \int d \tau \sqrt{-u^{\mu} u_{\mu}} \phi(z(\tau))
\end{aligned}
$$

where the current density $j(x ; z]$ is given by

$$
j(x ; z]=-\frac{e}{4 \pi} \int d \tau \sqrt{-g_{\mu \nu} u^{\mu} u^{\nu}} \frac{\delta^{4}(x-z(\tau))}{\sqrt{-g}}
$$

At this stage, $\tau$ is just a parameter of the worldline and not necessarily the proper time. We use an overdot to denote differentiation with respect to the worldline parameter $\tau$. For example, the 4 -velocity of a particle in Minkowski space $\left(g_{\mu \nu}=\eta_{\mu \nu}\right)$ is $u^{\alpha}=\frac{d z^{\alpha}}{d \tau}=\dot{z}^{\alpha}$. Because the particle is moving in a gravitational field, one should replace ordinary derivatives $d / d \tau$ by covariant derivatives $D / d \tau$ and so, for instance, the 4-acceleration is $\frac{D u^{\alpha}}{d \tau}=u^{\beta} \nabla_{\beta} u^{\alpha}$ and not $\dot{u}^{\alpha}=\frac{d u^{\alpha}}{d \tau}=u^{\beta} \partial_{\beta} u^{\alpha}$.

To facilitate easier computation it is customary to choose the initial density matrix to correspond to a factorized state of the system and environment. Physically, this means that all of the field modes have been uncorrelated with the particle by an instantaneous measurement at time $t_{i}$. Aside from issues about performing this measurement simultaneously in the spacetime, this choice is somewhat unphysical because, as explained in [15], an infinite amount of energy is required to uncorrelate all the modes of the environment (field) from the system (particle) at a particular instant of time. For instance, in models of quantum Brownian motion with an infinite number of environment oscillators the factorized initial state results in large transients of the diffusion coefficients appearing in the master equation for the reduced density matrix 15]. The transients appear as a result of the high frequency modes of the environment beginning to interact and correlate with the system just after the initial time. This recorrelation time lasts on the order of the inverse of the cut-off frequency used to regulate the divergences coming from oscillators of very high frequencies. For times much longer than this transient time the behavior due to the initial factorization is usually discounted. Other methods, including the preparation function method [16], allow for a somewhat more physical initial state by including certain system-environment correlations (e.g. system in thermal equilibrium with the environment at the initial time), but still seem to suffer from some of the problems associated with the factorized state [17].

Having said this we assume that there is a Cauchy hypersurface at the initial time $t_{i}$ such that the initial density matrix takes the factorized form

$$
\rho\left(z_{i}, \phi_{i} ; z_{i}^{\prime}, \phi_{i}^{\prime} ; t_{i}\right)=\rho_{S}\left(z_{i}, z_{i}^{\prime} ; t_{i}\right) \otimes \rho_{E}\left(\phi_{i}, \phi_{i}^{\prime} ; t_{i}\right)
$$

This simple form eases the manipulations for obtaining a description of the reduced particle dynamics.

After tracing out (a form of coarse-graining) the field variables from the density matrix the reduced density matrix for the system is given by 


$$
\begin{aligned}
& \rho_{r}\left(z_{f}, z_{f}^{\prime} ; t_{f}\right)=\int d \phi_{f} \rho\left(z_{f}, \phi_{f} ; z_{f}^{\prime}, \phi_{f} ; t_{f}\right) \\
& =\int d z_{i} d z_{i}^{\prime} \int_{z_{i}}^{z_{f}} \mathcal{D} z \int_{z_{i}^{\prime}}^{z_{f}^{\prime}} \mathcal{D} z^{\prime} \rho_{S}\left(z_{i}, z_{i}^{\prime} ; t_{i}\right) e^{\frac{i}{\hbar}\left(S_{S}[z]-S_{S}\left[z^{\prime}\right]\right)} \\
& \quad \times \int d \phi_{f} d \phi_{i} d \phi_{i}^{\prime} \int_{\phi_{i}}^{\phi_{f}} \mathcal{D} \phi \int_{\phi_{i}^{\prime}}^{\phi_{f}} \mathcal{D} \phi^{\prime} \rho_{E}\left(\phi_{i}, \phi_{i}^{\prime} ; t_{i}\right) e^{\frac{i}{\hbar}\left(S_{E}[\phi]+S_{i n t}[z, \phi]-S_{E}\left[\phi^{\prime}\right]-S_{i n t}\left[z^{\prime}, \phi^{\prime}\right]\right)} \\
& =\int d z_{i} d z_{i}^{\prime} \int_{z_{i}}^{z_{f}} \mathcal{D} z \int_{z_{i}^{\prime}}^{z_{f}^{\prime}} \mathcal{D} z^{\prime} \rho_{S}\left(z_{i}, z_{i}^{\prime} ; t_{i}\right) e^{\frac{i}{\hbar}\left(S_{S}[z]-S_{S}\left[z^{\prime}\right]\right)} F\left[z, z^{\prime}\right]
\end{aligned}
$$

The last line introduces the influence functional $F\left[z, z^{\prime}\right]$, which is given by

$$
F\left[z, z^{\prime}\right]=\int d \phi_{f} d \phi_{i} d \phi_{i}^{\prime} \int_{\phi_{i}}^{\phi_{f}} \mathcal{D} \phi \int_{\phi_{i}^{\prime}}^{\phi_{f}} \mathcal{D} \phi^{\prime} \rho_{E}\left(\phi_{i}, \phi_{i}^{\prime} ; t_{i}\right) e^{\frac{i}{\hbar}\left(S_{E}[\phi]+S_{i n t}[z, \phi]-S_{E}\left[\phi^{\prime}\right]-S_{i n t}\left[z^{\prime}, \phi^{\prime}\right]\right)}=e^{\frac{i}{\hbar} S_{i n f}\left[z, z^{\prime}\right]}
$$

and $S_{\text {inf }}$ is the influence action. In operator language $F$ is

$$
F\left[z, z^{\prime}\right]=\operatorname{Tr}_{E} \hat{U}_{E+i n t}\left(t_{f}, t_{i} ; z\right] \hat{\rho}_{E}\left(t_{i}\right) \hat{U}_{E+i n t}^{\dagger}\left(t_{f}, t_{i} ; z^{\prime}\right]
$$

where $\hat{U}_{E+i n t}\left(t_{f}, t_{i} ; z\right]$ is the evolution operator that evolves the environment variables through its interaction with the system. The influence functional can be interpreted as the overlap of the environment states evolved forward and backward in time while interacting with different particle trajectories, or simply as the ensemble average of the evolution operator $U_{E+\text { int }}[z]$ evolved backward in time under a different external source $z^{\prime}$, as can be seen by writing (2.9) as

$$
\begin{aligned}
& F\left[z, z^{\prime}\right]=\left\langle\hat{U}_{E+i n t}^{\dagger}\left[z^{\prime}\right] \hat{U}_{E+i n t}[z]\right\rangle_{e n s} \\
& \quad=\sum_{\alpha} \sum_{\alpha^{\prime}} \rho_{E, \alpha \alpha^{\prime}}\left(t_{i}\right)\left\langle\alpha^{\prime}\left|\hat{U}_{E+i n t}^{\dagger}\left[z^{\prime}\right] \hat{U}_{E+i n t}[z]\right| \alpha\right\rangle
\end{aligned}
$$

In the interaction picture, the time evolution operator is $\hat{U}_{E+i n t}\left(t_{f}, t_{i} ; z\right]=T e^{\frac{i}{\hbar} \hat{\phi}_{I} \cdot j[z]}$ where the $\cdot$ denotes spacetime integration so that for two functions, possibly tensors, $A\left(x^{\alpha}\right)$ and $B\left(x^{\beta}\right)$

$$
A \cdot B \equiv \int_{x_{i}^{0}}^{x_{f}^{0}} d x^{0} \int d^{3} x \sqrt{-g} A(x) B(x)
$$

Assuming that the initial state of the field is Gaussian, the influence functional can be calculated exactly giving

$$
F\left[z, z^{\prime}\right]=e^{-\frac{1}{4 \hbar} j^{-} \cdot\left(16 \pi^{2} G_{H}\right) \cdot j^{-}+\frac{i}{\hbar} j^{-} \cdot\left(16 \pi^{2} G_{r e t}\right) \cdot j^{+}}
$$

for the influence functional and

$$
\begin{aligned}
& S_{I F}\left[z, z^{\prime}\right] \stackrel{\text { def }}{=}-i \hbar \ln F\left[z, z^{\prime}\right] \\
& =\frac{i}{4} j^{-} \cdot\left(16 \pi^{2} G_{H}\right) \cdot j^{-}+j^{-} \cdot\left(16 \pi^{2} G_{r e t}\right) \cdot j^{+}
\end{aligned}
$$

for the influence action. The difference and semi-sum current densities are defined as

$$
\begin{aligned}
& j^{-}=j[z]-j\left[z^{\prime}\right] \\
& j^{+}=\frac{j[z]+j\left[z^{\prime}\right]}{2}
\end{aligned}
$$

and the Hadamard $G_{H}$ and retarded Green's functions $G_{\text {ret }}$ are

$$
\begin{aligned}
& G_{H}\left(x, x^{\prime}\right)=\left\langle\left\{\hat{\phi}_{I}(x), \hat{\phi}_{I}\left(x^{\prime}\right)\right\}\right\rangle-2\left\langle\hat{\phi}_{I}(x)\right\rangle\left\langle\hat{\phi}_{I}\left(x^{\prime}\right)\right\rangle \\
& G_{r e t}\left(x, x^{\prime}\right)=i \theta_{+}(x, \Sigma)\left\langle\left[\hat{\phi}_{I}(x), \hat{\phi}_{I}\left(x^{\prime}\right)\right]\right\rangle
\end{aligned}
$$

The $\langle\cdots\rangle=\operatorname{Tr}_{E} \hat{\rho}_{E}(\cdots)$ denotes quantum expectation values in the Gaussian initial state $\hat{\rho}_{E}$ of the environment. The step function $\theta_{+}(x, \Sigma)$ appearing in $G_{r e t}$ equals one in the future of the point $x^{\prime}$ and zero otherwise. Here, $\Sigma$ is a space-like hypersurface containing $x^{\prime}$. Had the initial state contained non-Gaussian contributions, one would have many additional terms involving cubic and higher powers of the coupling. Likewise for nonlinear interactions (e.g. $\left.\sim e j[z] \cdot \phi^{n}\right)$. Assuming that the coupling is small and that non-Gaussianities are also small one can still use (2.12) and (2.13) as a lowest order approximation. 


\section{B. Semi-Classical Particle Dynamics}

The reduced density matrix for the particle is now written as

$$
\begin{aligned}
& \rho_{r}\left(z_{f}, z_{f}^{\prime} ; t_{f}\right) \\
& \quad=\int d z_{i} d z_{i}^{\prime} \int_{z_{i}}^{z_{f}} \mathcal{D} z \int_{z_{i}^{\prime}}^{z_{f}^{\prime}} \mathcal{D} z^{\prime} \rho_{S}\left(z_{i}, z_{i}^{\prime} ; t_{i}\right) e^{\frac{i}{\hbar} S_{C G E A}\left[z, z^{\prime}\right]}
\end{aligned}
$$

where the coarse-grained effective action (CGEA) is defined as

$$
\begin{aligned}
& S_{C G E A}\left[z, z^{\prime}\right] \\
& =S_{S}[z]-S_{S}\left[z^{\prime}\right]+S_{I F}\left[z, z^{\prime}\right] \\
& =S_{S}[z]-S_{S}\left[z^{\prime}\right]+j^{-} \cdot\left(16 \pi^{2} G_{r e t}\right) \cdot j^{+} \\
& \quad+\frac{i}{4} j^{-} \cdot\left(16 \pi^{2} G_{H}\right) \cdot j^{-}
\end{aligned}
$$

At this point it is worth mentioning that the magnitude of the influence functional decays rapidly for two largely separated histories since it is Gaussian (to lowest order) in $z^{-}=z-z^{\prime}$

$$
\begin{aligned}
& \left|F\left[z, z^{\prime}\right]\right|=e^{-\frac{1}{4 \hbar} j^{-} \cdot\left(16 \pi^{2} G_{H}\right) \cdot j^{-}} \\
& =\exp -\frac{e^{2}}{4 \hbar} \int d \tau \int d \tau^{\prime} z_{-}^{\alpha} \frac{\delta j^{-}}{\delta z_{-}^{\alpha}} G_{H}\left(z_{+}^{\mu}, z_{+}^{\mu^{\prime}}\right) \frac{\delta j^{-}}{\delta z_{-}^{\alpha^{\prime}}} z_{-}^{\alpha^{\prime}} \\
& \quad+\mathcal{O}\left(z_{-}^{4}\right)
\end{aligned}
$$

Here, and in the following, $z^{\alpha} \equiv z^{\alpha}(\tau)$ and $z^{\alpha^{\prime}} \equiv z^{\alpha}\left(\tau^{\prime}\right)$ so that an unprimed (primed) index refers to that component of a tensor field or coordinate evaluated at proper time $\tau\left(\tau^{\prime}\right)$ and

$$
\begin{aligned}
& z_{\mu}^{-}=z_{\mu}-z_{\mu}^{\prime} \\
& z_{\mu}^{+}=\frac{z_{\mu}+z_{\mu}^{\prime}}{2}
\end{aligned}
$$

The norm of $F$ is equal to the norm of the decoherence functional (see below), which is a measure of how much the particle's worldline is decohered. So, if the quantum fluctuations of the field (environment) provide a strong enough mechanism for decoherence (this should be checked on a case by case basis and will be assumed true in the cases under study here) then we are justified in expanding the CGEA about the classical trajectory $\bar{z}^{\mu}$. Doing so gives

$$
S_{C G E A}\left[z, z^{\prime}\right]=\left.\int d \tau^{\prime} z_{-}^{\alpha^{\prime}} \frac{\delta S_{C G E A}}{\delta z_{-}^{\alpha^{\prime}}}\right|_{z=z^{\prime}=\bar{z}}+\mathcal{O}\left(z_{-}^{2}\right)
$$

Using this expression in the reduced density matrix and doing a stationary phase approximation gives the equations of motion for the classical worldline $\bar{z}$

$$
\left.\frac{\delta S_{C G E A}}{\delta z_{-}^{\mu}(\tau)}\right|_{z=z^{\prime}=\bar{z}}=0
$$

Evaluating the functional derivative using, for some test function $f(x)$,

$$
\begin{aligned}
& \frac{\delta}{\delta z_{-}^{\mu}(\tau)} \int d^{4} x \sqrt{-g} j^{-}(x ; z] f(x) \\
& \quad=\frac{e}{4 \pi}\left(\frac{D u_{\mu}}{d \tau}+w_{\mu}{ }^{\nu}[z] \nabla_{z^{\nu}}\right) f(z) \\
& \quad \stackrel{\text { def }}{=} \frac{e}{4 \pi} \vec{w}_{\mu}[z] f(z)
\end{aligned}
$$

where $w_{\mu}^{\nu}=g_{\mu}{ }^{\nu}+u_{\mu} u^{\nu}$ and $u^{\alpha} u_{\alpha}=-1$ (proper-time gauge), gives

$$
m_{0} \frac{D \bar{u}_{\mu}}{d \tau}=e \vec{w}_{\mu}[\bar{z}] \phi_{r e t}(\bar{z})
$$

where $\phi_{\text {ret }}$ is the retarded field

$$
\phi_{\text {ret }}(x)=e \int d \tau^{\prime} G_{r e t}\left(x, z^{\alpha^{\prime}}\right)
$$

The right side of (2.25) is the self-force on the particle arising from the radiation reaction. Furthermore, the vector operator $\vec{w}_{\mu}$ contains the particle's acceleration implying that the particle moves with an effective time-dependent mass equal to $m_{0}-e \phi_{\text {ret }}(\bar{z})$. (See [19] for a discussion of evaporating scalar charges based on this time-dependent effective mass.) Provided there is strong enough decoherence to suppress the quantum fluctuations of the worldline, the equations of motion for the quantum expectation value $\left\langle\hat{z}^{\mu}\right\rangle$ are the same as in (2.25) at tree-level in both the particle and the field [10]. Unfortunatley, 2.25) is problematic since the self-force diverges on the worldline of the point particle. This divergence can be traced to the singular coincidence limit of the retarded Green's function $\lim _{\tau^{\prime} \rightarrow \tau} G_{r e t} \rightarrow \infty$ and results from the point particle assumption. We will discuss how to treat this problem in Sec. III by adopting an effective field theory point of view.

\section{Stochastic Semi-Classical Particle Dynamics}

We have made the assumption that the quantum fluctuations of the worldline are strongly suppressed by the decoherence due to the quantum field. Even under strong decoherence when the classical trajectory is well-defined, the quantum fluctuations of the field can still influence the classical motion of the particle through the particlefield coupling $S_{\text {int }}$. They may show up as classical stochastic forces on the particle. In this section we shall show how this comes about using the influence functional for the reduced density matrix.

We start by invoking the relation

$$
e^{-\frac{1}{4 \hbar} j^{-} \cdot\left(16 \pi^{2} G_{H}\right) \cdot j^{-}}=N \int \mathcal{D} \xi(x) e^{-\frac{1}{\hbar} \xi \cdot G_{H}^{-1} \cdot \xi-\frac{4 \pi i}{\hbar} \xi \cdot j^{-}}
$$


where $N$ is a normalization factor that is independent of the worldline coordinates and $\xi(x)$ is some auxiliary field. Using this relation, the influence functional (2.12) can be written as

$F\left[z, z^{\prime}\right]=N \int \mathcal{D} \xi(x) e^{-\frac{1}{\hbar} \xi \cdot G_{H}^{-1} \cdot \xi+\frac{i}{\hbar} j^{-} \cdot\left(-4 \pi \xi+16 \pi^{2} G_{r e t} \cdot j^{+}\right)}$

where $\xi(x)$ appears as an auxiliary function, which can be interpreted as a classical stochastic or noise field $[10$, 18] with an associated Gaussian probability distribution functional

$$
P_{\xi}[\xi(x)]=e^{-\frac{1}{\hbar} \xi \cdot G_{H}^{-1} \cdot \xi}
$$

The fact that this is Gaussian is a direct consequence of taking an initial Gaussian state for the quantum field. With respect to $P_{\xi}[\xi]$ this implies that $\xi$ has zero-mean and its correlator is proportional to the Hadamard function encoding the information about the fluctuations in the quantum field [37].

$$
\begin{aligned}
\langle\xi(x)\rangle_{\xi} & =0 \\
\left\langle\left\{\xi(x), \xi\left(x^{\prime}\right)\right\}\right\rangle_{\xi} & =\hbar G_{H}\left(x, x^{\prime}\right)
\end{aligned}
$$

where $\langle\ldots\rangle_{\xi}=N \int \mathcal{D} \xi P_{\xi}(\ldots)$.

Now the reduced density matrix (2.7) becomes

$$
\rho_{r}\left(z_{f}, z_{f}^{\prime} ; t_{f}\right)=N \int d z_{i} d z_{i}^{\prime} \int_{z_{i}}^{z_{f}} \mathcal{D} z \int_{z_{i}^{\prime}}^{z_{f}^{\prime}} \mathcal{D} z^{\prime} \rho_{S}\left(z_{i}, z_{i}^{\prime} ; t_{i}\right) \int \mathcal{D} \xi P_{\xi}[\xi] e^{\frac{i}{\hbar} S_{S E A}\left[z, z^{\prime} ; \xi\right]}
$$

where the stochastic effective action (SEA) is defined as

$$
S_{S E A}\left[z, z^{\prime} ; \xi\right]=S_{S}[z]-S_{S}\left[z^{\prime}\right]+j^{-} \cdot\left(-4 \pi \xi+16 \pi^{2} G_{r e t} \cdot j^{+}\right)=S_{C G E A}^{R}\left[z, z^{\prime}\right]-(4 \pi) \xi \cdot j^{-}
$$

and $S_{C G E A}^{R}$ is the real part of the CGEA. As before, if the worldline is strongly decohered by the quantum fluctuations of the environment then we are justified in expanding the SEA around the classical solution

$$
S_{S E A}\left[z, z^{\prime} ; \xi\right]=-\int d \tau^{\prime} z_{-}^{\alpha^{\prime}} \eta_{\alpha^{\prime}}\left[z^{+}\right]+\left.\frac{1}{2} \int d \tau^{\prime} \int d \tau^{\prime \prime} z_{-}^{\alpha^{\prime}} z_{-}^{\alpha^{\prime \prime}} \frac{\delta^{2} S_{C G E A}^{R}}{\delta z_{+}^{\alpha^{\prime}} \delta z_{+}^{\alpha^{\prime \prime}}}\right|_{z=z^{\prime}=\bar{z}}+\mathcal{O}\left(z_{-}^{3}\right)
$$

where we have used (2.23), expanded $j^{-}$in powers of $z_{-}$ and defined the stochastic force

$$
\eta_{\mu}[z]=e \vec{w}_{\mu}[z] \xi(z)
$$

Putting (2.34) into the reduced density matrix and using the stationary phase approximation gives the stochastic equations of motion for the worldline fluctuations $z_{-}^{\mu}=$ $\tilde{z}^{\mu} \equiv z^{\mu}-\bar{z}^{\mu}$

$$
\left.\int d \tau^{\prime} \tilde{z}^{\alpha^{\prime}} \frac{\delta^{2} S_{C G E A}^{R}}{\delta \bar{z}^{\mu} \delta \bar{z}^{\alpha^{\prime}}}\right|_{z=z^{\prime}=\bar{z}}=\eta_{\mu}[\bar{z}]
$$

It should be emphasized that this equation describes the evolution of small perturbations $\tilde{z}$ around the semiclassical trajectory that arise from the stochastic manifestation $\eta$ of the quantum field fluctuations. We can obtain a stochastic version of the ALD equation (2.25) if we add the equations of motion for the classical dynamics

$$
0=\left.\frac{\delta S_{C G E A}^{R}}{\delta z_{-}^{\mu}}\right|_{z=z^{\prime}=\bar{z}}
$$

to the left side. Then we may write

$$
\left.\frac{\delta S_{C G E A}^{R}}{\delta z_{-}^{\mu}}\right|_{z_{-}=0}=\eta_{\mu}[z]
$$

Evaluating this functional derivative gives the stochastic semi-classical particle dynamics for the full worldline $z_{\mu}=\bar{z}_{\mu}+\tilde{z}_{\mu}$

$$
m_{0} \frac{D u_{\mu}}{d \tau}=e \vec{w}_{\mu}[z] \phi_{r e t}(z)+\eta_{\mu}[z]
$$

Notice that both the deterministic and the stochastic components of the self-force can push the particle away from its geodesic motion with respect to a fixed background spacetime. However, we should keep in mind that this equation is really only valid to linear order in the fluctuations $\tilde{z}$ since higher order terms will correspond to quantum corrections that we have been neglecting. Of course, we could have obtained (2.39) by using a stationary phase approximation in the reduced density matrix (2.32) without having expanded around the classical trajectory. However, we believe that the validity of (2.39) to linear order in the fluctuations would not have been as transparent.

The stochastic correlation functions of the force $\eta_{\mu}$ can be evaluated using the knowledge of the $\xi$ correlators above. As was commented in the previous paragraph we must evaluate these correlation functions along the classical trajectory $\bar{z}$ to be consistent with the linearization. 
The mean of the stochastic force is zero

$$
\left\langle\eta_{\mu}[\bar{z}]\right\rangle_{\xi}=e \vec{w}_{\mu}[\bar{z}]\langle\xi(\bar{z})\rangle_{\xi}=0
$$

and the symmetric two-point function of the stochastic force is

$$
\left\langle\left\{\eta_{\mu}[\bar{z}], \eta_{\mu^{\prime}}[\bar{z}]\right\}\right\rangle_{\xi}=\hbar e^{2} \vec{w}_{(\mu}[\bar{z}] \vec{w}_{\left.\mu^{\prime}\right)}[\bar{z}] G_{H}\left(\bar{z}^{\alpha}, \bar{z}^{\alpha^{\prime}}\right)
$$

This shows that the noise $\eta_{\mu}$ is multiplicative, colored and depends on the particle's initial conditions through the classical trajectory. The noise correlator also generically depends on the field's initial conditions as is seen by the appearance of $G_{H}$ in the equation. The Hadamard function $G_{H}$ does not vanish on a space-like hypersurface implying that the quantum correlations in the environment are non-local. However, the noise correlator above is evaluated on time-like separated points only. For equal proper times $\tau^{\prime}=\tau$ the Hadamard function diverges so a suitable regularization procedure must be used in order to make sense of (2.41) near coincidence.

In the next two sections we describe a new method introduced in [10] for regulating the ultraviolet divergences in the retarded Green's function. We introduce a high energy scale in the quantum field below which the low energy point particle dynamics is expected to be valid. For long times as compared to the inverse of the high energy scale, we invoke a quasi-local expansion in order to obtain the relevant contributions to the particle's motion. Our work is a generalization of [10] to curved spacetime. We follow Poisson's description of scalar radiation reaction in curved spacetime and use units where $c=G=1$. (We change the particle physics notation in [10] $(+,-,-$, -) to the MTW notation $(-,+,+,+)$ of $[\underline{6}]$.)

\section{REGULARIZATION OF THE RETARDED GREEN'S FUNCTION}

Before we discuss regularizing the retarded Green's function it will be beneficial to introduce some notation. Following [ 6$]$ we define the step function $\theta_{+}(x, \Sigma)$ to be 1 for any point $x$ to the future of a space-like hypersurface $\Sigma$ and 0 otherwise. This is a generalization of the ordinary step function to curved spacetime. Using this, one can define the following distributions

$$
\begin{aligned}
\delta_{+}\left(\sigma\left(x, x^{\prime}\right)\right) & =\theta_{+}(x, \Sigma) \delta\left(\sigma\left(x, x^{\prime}\right)\right) \\
\theta_{+}\left(-\sigma\left(x, x^{\prime}\right)\right) & =\theta_{+}(x, \Sigma) \theta\left(-\sigma\left(x, x^{\prime}\right)\right)
\end{aligned}
$$

where $\sigma\left(x, x^{\prime}\right)$ is Synge's world function along the (unique) geodesic linking $x=z(\tau)$ and $x^{\prime}=z\left(\tau^{\prime}\right)$. Taking the lightcone centered on $x^{\prime}$, with $x^{\prime}$ on the space-like hypersurface $\Sigma$, it follows that $\delta_{+}(\sigma)$ has support along the forward lightcone only while $\theta_{+}(-\sigma)$ is one in the causal future of $x^{\prime}$ and vanishes everywhere else.

In (2.16) the retarded Green's function is calculated from the commutator of the interaction picture field $\hat{\phi}_{I}$.
Recall that $\hat{\phi_{I}}$ evolves under the free dynamics so that

$$
\left(\square-\xi_{R} R\right) \phi_{I}=0
$$

and so is the homogeneous solution to the full field equation in the Heisenberg picture (satisfying the same initial conditions)

$$
\left(\square-\xi_{R} R\right) \phi_{H}=j[z]
$$

where $\hat{\phi}_{H}=\hat{\phi}_{I}+\hat{1} G_{r e t} \cdot j[z]$ for a classical source. Because the source is classical only the first term in the solution gives a contribution to the commutator and hence to the retarded Green's function

$$
G_{r e t} \sim\left\langle\left[\hat{\phi}_{H}(x), \hat{\phi}_{H}\left(x^{\prime}\right)\right]\right\rangle=\left\langle\left[\hat{\phi}_{I}(x), \hat{\phi}_{I}\left(x^{\prime}\right)\right]\right\rangle
$$

Finally, since the commutator is state-independent it follows that $G_{r e t}$ for the quantum field $\hat{\phi}_{H}$ and for the corresponding classical field $\phi$ are the same.

\section{A. Hadamard Expansion}

This allows us to invoke Hadamard's ansatz for which the retarded Green's function for a scalar field in a curved $3+1$ dimensional spacetime has the form $[6]$

$$
\begin{aligned}
& G_{r e t}\left(x, x^{\prime}\right) \\
& =\Delta^{1 / 2}\left(x, x^{\prime}\right) \delta_{+}\left(\sigma\left(x, x^{\prime}\right)\right)+V\left(x, x^{\prime}\right) \theta_{+}\left(-\sigma\left(x, x^{\prime}\right)\right)
\end{aligned}
$$

which is the sum of a "direct" part (proportional to $\delta_{+}$) and a "tail" part (proportional to $\theta_{+}(-\sigma)$ ). Notice that the appearance of $\delta_{+}$and $\theta_{+}$ensures that $G_{r e t}$ has the correct causal structure. In order for this ansatz to be valid, the points $x$ and $x^{\prime}$ must be connected by a unique geodesic. Otherwise, ambiguities arise from the appearance of caustics when parallel propagating a tensor field from $x^{\prime}$ to $x$ or vice versa. This will be assumed in the following. The function $\Delta\left(x, x^{\prime}\right)$ is the van Vleck determinant and the function $V\left(x, x^{\prime}\right)$ satisfies the homogeneous Klein-Gordon equation in curved spacetime

$$
\left(\square_{g}-\xi_{R} R\right) V\left(x, x^{\prime}\right)=0
$$

with boundary data determined by the restriction of $V$ to the forward lightcone, denoted by $\tilde{V}$. The restriction of $V$ is found by solving

$$
\tilde{V}_{, \alpha} \sigma^{\alpha}+\frac{1}{2}\left(\sigma_{\alpha}^{\alpha}-2\right) \tilde{V}=\frac{1}{2}\left\{\left(\square_{g}-\xi_{R} R\right) \Delta^{1 / 2}\right\}_{\sigma=0}
$$

Knowing all of the light-like geodesics emanating into the future from $x^{\prime}$, one can integrate this equation to construct $\tilde{V}$ on the light-cone and hence $V$ inside the light-cone. Of course, this just solves for $V\left(x, x^{\prime}\right)$ for any $x$ to the causal future of $x^{\prime}$ and must be solved again for different values of $x^{\prime}$. This makes determining $V$ very 
difficult and tedious for generic spacetimes. However, if the spacetime possesses enough symmetry (e.g. de Sitter, some FRW cosmologies) then $V$ can be constructed relatively easily [19].

From the mean and stochastic equations of motion (2.25) and (2.39) it is necessary to compute the restriction of $G_{\text {ret }}$ to the particle's worldline

$$
\begin{gathered}
G_{r e t}\left(z^{\alpha}, z^{\alpha^{\prime}}\right)=\Delta^{1 / 2}\left(z^{\alpha}, z^{\alpha^{\prime}}\right) \delta_{+}\left(\sigma\left(z^{\alpha}, z^{\alpha^{\prime}}\right)\right) \\
+V\left(z^{\alpha}, z^{\alpha^{\prime}}\right) \theta_{+}\left(-\sigma\left(z^{\alpha}, z^{\alpha^{\prime}}\right)\right)
\end{gathered}
$$

Using the Hadamard ansatz and 2.26) it is easy to see that for two (time-like separated) points on the particle worldline, $\delta_{+}(\sigma)$ has support only at coincidence, when $\tau^{\prime}=\tau$. The contribution from the direct term is then

$$
\begin{aligned}
& \int_{\tau_{i}}^{\tau_{f}} d \tau^{\prime} \Delta^{1 / 2}\left(z^{\alpha}, z^{\alpha^{\prime}}\right) \theta_{+}\left(z^{\alpha}, S\right) \delta\left(\sigma\left(z^{\alpha}, z^{\alpha^{\prime}}\right)\right) \\
= & \int_{\tau_{i}}^{\tau} d \tau^{\prime} \Delta^{1 / 2}\left(z^{\alpha}, z^{\alpha^{\prime}}\right) \delta\left(\tau^{\prime}-\tau\right)\left(\frac{d \sigma}{d \tau}\right)_{\tau^{\prime}=\tau}^{-1}
\end{aligned}
$$

This expression is ultraviolet divergent since $d \sigma / d \tau$ evaluates to zero for $\tau^{\prime}=\tau$ and so the retarded field is not well-defined (in fact, it is infinite) when evaluated at the location of the point particle. This feature is not limited to just a quantum field as it appears even for a classical field since they share the same retarded Green's function.

The presence of this divergence suggests that a regularization procedure is needed to render the equations of motion (2.25) and (2.39) finite. Several procedures have been proposed in the literature for doing this. In [5, 6], the retarded field is evaluated near the worldline so that the divergence can be tracked in the limit that one approaches the worldline. This divergence renormalizes the particle's mass. Writing the retarded field in terms of functions singular $\phi_{S}$ and regular $\phi_{R}$ on the worldline allows, after calculating their gradient and expanding them near the worldline, for the self-force to be evaluated unambiguously along the worldline. This method can also be used for electromagnetic and gravitational self-force calculations. In another method developed in [20, 21, 22, 23, 24], a mode-sum regularization procedure is used in which the self-force is decomposed in terms of the multipole moments of the field. From this one subtracts the moments of the singular part of the self-force and resums over the multipoles to obtain a self-force that is finite on the worldline. In 25] the "massive field approach" is used in which an auxiliary massive field is introduced to subtract away the ultraviolet divergence from the massless field. This procedure is similar to Pauli-Villars type regularization and has also been used in [13] to regularize the direct term in $G_{\text {ret }}$.

Other regularization methods have been used for the radiation reaction of non-relativistic particles without gravity present [26]. Notable of these include the extended-charge models of [27] in which the particle is described as a rigid volume with a non-zero size. Unfortunately, this type of prescription cannot be extended to relativistic models since rigid bodies are incompatible with relativity [38]. Furthermore, the successes of quantum field theory (e.g. as applied to the scattering of point particles), has proved to be an adequate description of the low-energy effective dynamics of point particles to $\sim \mathrm{TeV}$ scales. It seems unnecessary, then, to introduce non-trivial structure to the particle to study the self-force.

\section{B. Regularization motivated by Effective Field Theory}

With this consideration in mind we introduce yet another regularization for the self-force motivated by [10]. All theories for the fundamental interactions (e.g. QED, electroweak theory, QCD) can be interpreted as effective theories, in that they are are low energy limits of a more complete theory valid at higher energies, yet they provide an excellent description of low energy phenomena, at least for renormalizable theories, without requiring the details of the complete theory. For energies much higher than the energy scale of the effective theory, new physics is likely to become important.

In this spirit we introduce a regulator $\Lambda$ for the field. Above the energy scale of this regulator, $E_{\Lambda}$, new physics is assumed to occur. For energies much lower than $E_{\Lambda}$, the dynamics (2.25) and (2.39) will be sufficiently accurately described by using a regulated quantum field. This approach has been taken in 10] in deriving the AbrahamLorenz-Dirac (ALD) equations in flat spacetime. It is easily extended to motions in a curved spacetime since the direct part of the retarded Green's function is a local quantity on the worldline.

Regularization is achieved here by choosing any suitably smooth function to approximate $\delta_{+}$for large values of the regulator $\Lambda$. Following [10] we replace $\delta_{+}$with

$$
\begin{aligned}
\delta_{+}\left(\sigma\left(z^{\alpha}, z^{\alpha^{\prime}}\right)\right) & \rightarrow \theta_{+}\left(-\sigma\left(z^{\alpha}, z^{\alpha^{\prime}}\right)\right) \sqrt{\frac{8}{\pi}} \Lambda^{2} e^{-2 \Lambda^{4} \sigma^{2}\left(z, z^{\prime}\right)} \\
& =\theta_{+}\left(-\sigma\left(z^{\alpha}, z^{\alpha^{\prime}}\right)\right) g_{r e t}^{\Lambda}\left(z^{\alpha}, z^{\alpha^{\prime}}\right)
\end{aligned}
$$

In the limit of infinitely large $\Lambda$ we have that $\theta_{+} g_{\text {ret }}^{\Lambda}$ approaches $\delta_{+}$. The integral of the direct part of $G_{\text {ret }}$ (3.10) receives a contribution from $\delta_{+}$at coincidence implying that $\sigma=0$. The function $g_{\text {ret }}^{\Lambda}$ is smooth but approximates $\delta_{+}$well only if $\Lambda^{2} \sigma \gg 1$. This approximation will not hold if $\sigma$ is identically zero. Nevertheless, we will assume that $\sigma$ is small and approaching zero but $\Lambda$ is such that $\Lambda^{2} \sigma \gg 1$ still holds. This separation of scales allows us to do a quasi-local expansion below in which the self-force will be expanded near coincidence.

Making the replacement (3.11) in $G_{r e t}$ gives the regulated Green's function 


$$
G_{r e t}^{\Lambda}\left(z^{\alpha}, z^{\alpha^{\prime}}\right)=\theta_{+}\left(-\sigma\left(z^{\alpha}, z^{\alpha^{\prime}}\right)\right)\left\{\Delta^{1 / 2}\left(z^{\alpha}, z^{\alpha^{\prime}}\right) g_{r e t}^{\Lambda}\left(z^{\alpha}, z^{\alpha^{\prime}}\right)+V\left(z^{\alpha}, z^{\alpha^{\prime}}\right)\right\}=\theta_{+}(-\sigma)\left\{\Delta^{1 / 2} g_{r e t}^{\Lambda}+V\right\}
$$

where an abbreviated notation has been used on the far right side to ease the appearance of later expressions. Also, it should be clear that in the limit $\Lambda \rightarrow \infty$ that $G_{r e t}^{\Lambda} \rightarrow G_{r e t}$.

Putting $G_{r e t}^{\Lambda}$ into (2.25) gives

$$
m_{0} \frac{D \bar{u}_{\mu}}{d \tau}=e^{2}\left(\frac{D \bar{u}_{\mu}}{d \tau}+w_{\mu}^{\nu}[\bar{z}] \nabla_{\bar{z}^{\nu}}\right) \int_{\tau_{i}}^{\tau_{f}} d \tau^{\prime} \theta\left(\tau-\tau^{\prime}\right)\left\{\Delta^{1 / 2} g_{r e t}^{\Lambda}+V\right\}
$$

where the quantities in the integral are evaluated along the classical trajectory $\bar{z}$. Passing the derivative through the integral gives

$$
m_{0} \frac{D \bar{u}_{\mu}}{d \tau}=e \frac{D \bar{u}_{\mu}}{d \tau} \phi_{r e t}(\bar{z})+e^{2} w_{\mu}^{\nu}[\bar{z}]\left\{\left[\Delta^{1 / 2} g_{r e t}^{\Lambda}+V\right]\left[\nabla_{\bar{z}^{\nu}}\left(\tau-\tau^{\prime}\right)\right]+\int_{\tau_{i}}^{\tau} d \tau^{\prime} \nabla_{\bar{z}^{\nu}}\left(\Delta^{1 / 2} g_{r e t}^{\Lambda}+V\right)\right\}
$$

where the $[\ldots]$ denotes the coincidence limit $\tau^{\prime} \rightarrow \tau$ of the quantity it contains. This notation looks the same when denoting a functional of a function (e.g. $\left.w_{\mu}^{\nu}[z]\right)$ but the context should be clear. From Appendix A one can show that

$$
\left[\nabla_{\bar{z}^{\nu}}\left(\tau-\tau^{\prime}\right)\right]=-\left[\nabla_{\nu}\left(\bar{\sigma}^{\alpha} \bar{u}_{\alpha}\right)\right]=-\bar{u}_{\nu}
$$

and since $w_{\mu}^{\nu}[\bar{z}]$ projects vectors onto a direction orthogonal to the mean 4-velocity then $w_{\mu}{ }^{\nu}[\bar{z}]\left[\nabla_{\bar{z}^{\nu}}\left(\tau-\tau^{\prime}\right)\right]$ gives no contribution leaving

$$
\begin{aligned}
m_{0} \frac{D \bar{u}_{\mu}}{d \tau} & =e^{2} \frac{D \bar{u}_{\mu}}{d \tau} \phi_{r e t}(\bar{z})+e^{2} w_{\mu}^{\nu}[\bar{z}] \int_{\tau_{i}}^{\tau} d \tau^{\prime} \nabla_{\bar{z}^{\nu}}\left(\Delta^{1 / 2} g_{r e t}^{\Lambda}+V\right) \\
& =e^{2} \frac{D \bar{u}_{\mu}}{d \tau} \phi_{r e t}(\bar{z})+e^{2} w_{\mu}^{\nu}[\bar{z}] \int_{\tau_{i}}^{\tau} d \tau^{\prime}\left\{\left(\nabla_{\bar{z}^{\nu}} \Delta^{1 / 2}\right) g_{r e t}^{\Lambda}+\Delta^{1 / 2} \nabla_{\bar{z}^{\nu}} g_{r e t}^{\Lambda}+\nabla_{\bar{z}^{\nu}} V\right\}
\end{aligned}
$$

It will be convenient for later manipulations to define the tail term as the integral over the past history of the gradient of $V$,

$$
\phi_{\nu}^{\text {tail }}(\bar{z}(\tau))=e \int_{\tau_{i}}^{\tau} d \tau^{\prime} \nabla_{\bar{z}^{\nu}} V
$$

The tail term will turn out to be responsible for the nonMarkovian and history-dependent dynamics of the particle's evolution. Consequently, this presents a significant source of difficulty when trying to determine the particle's motion since one has to know the entire past history of the particle's worldline in order to make predictions about its current position and speed.

\section{EFFECT OF RADIATION REACTION ON PARTICLE TRAJECTORY}

In order to simplify some of the difficulties of solving the nonlinear integro-differential equation (3.16) we uti- lize the effective theory viewpoint discussed in the previous section. Since $g_{\text {ret }}^{\Lambda}$ is strongly peaked around $\sigma=0$ then the contributions to the self-force will be largest when $\tau^{\prime} \approx \tau$. We therefore introduce a quasi-local expansion by expanding $\theta_{+} \Delta^{1 / 2} g_{\text {ret }}^{\Lambda}$ around coincidence $\tau^{\prime}=\tau$. This yields a well-defined description of the selfforce in terms of relevant and irrelevant quantities. The relevant terms are those that either renormalize certain parameters of the particle or otherwise affect the particle dynamics when $\Lambda$ goes to infinity while the irrelevant terms are those that give no contribution in the same limit.

\section{A. Quasilocal Expansion}

We begin by expanding the van Vleck determinant and $g_{\text {ret }}^{\Lambda}$ for small values of $\sigma\left(z^{\alpha}, z^{\alpha^{\prime}}\right)$. Following [6] the expansion of the square root of the van Vleck determinant around $\sigma, \sigma^{\alpha}=0$ is

$$
\Delta^{1 / 2}\left(\bar{z}^{\alpha}, \bar{z}^{\alpha^{\prime}}\right)=1+\frac{1}{12} R_{\alpha \beta}(\bar{z}) \sigma^{\alpha} \sigma^{\beta}-\frac{1}{36} R_{\alpha \beta ; \gamma} \sigma^{\alpha} \sigma^{\beta} \sigma^{\gamma}+\ldots
$$


where the ... denotes terms of higher order in the expansion. The covariant derivative with respect to the worldline coordinate of the above quantity is

$$
\nabla_{\bar{z}^{\nu}} \Delta^{1 / 2}\left(\bar{z}^{\alpha}, \bar{z}^{\alpha^{\prime}}\right)=\frac{1}{6} R_{\alpha \beta}(\bar{z}) \sigma_{; \nu}^{\alpha} \sigma^{\beta}+\frac{1}{12} R_{\alpha \beta ; \nu}(\bar{z}) \sigma^{\alpha} \sigma^{\beta}+\ldots
$$

Lastly, $\sigma_{; \nu}^{\alpha}$ can be expanded around small $\sigma$ to give

$$
\sigma_{; \nu}^{\alpha}=g_{\nu}^{\alpha}-\frac{1}{3} R_{\gamma \nu \delta}^{\alpha}(\bar{z}) \sigma^{\gamma} \sigma^{\delta}+\ldots
$$

so that the gradient of $\Delta^{1 / 2}$ becomes

$$
\nabla_{\bar{z}^{\nu}} \Delta^{1 / 2}\left(\bar{z}^{\alpha}, \bar{z}^{\alpha^{\prime}}\right)=\frac{1}{6} R_{\nu \alpha}(\bar{z}) \sigma^{\alpha}-\frac{1}{18} R_{\alpha \beta \nu ; \lambda}^{\lambda}(\bar{z}) \sigma^{\alpha} \sigma^{\beta}+\ldots
$$

Next, we expand these quantities and $g_{r e t}^{\Lambda}$ around $\tau^{\prime}=\tau+s$ with $s$ such that $\Lambda s \gg 1$. The expansions of some relevant quantities involving the world function $\sigma$ are (see Appendix A for details)

$$
\begin{aligned}
s & =\tau^{\prime}-\tau \\
\sigma\left(\tau, \tau^{\prime}\right) & =-\frac{1}{2} s^{2}+\mathcal{O}\left(s^{4}\right) \\
\sigma_{\mu}(\tau, s) & =-s u_{\mu}(\tau)-\frac{s^{2}}{2} \frac{D u_{\mu}(\tau)}{d \tau}-\frac{s^{3}}{6} \frac{D^{2} u_{\mu}(\tau)}{d \tau^{2}}+\mathcal{O}\left(s^{4}\right)
\end{aligned}
$$

Using these results, the function $g_{r e t}^{\Lambda}$ is

$$
g_{\text {ret }}^{\Lambda}=\sqrt{\frac{8}{\pi}} \Lambda^{2} e^{-2 \Lambda^{4} \sigma^{2}}=\sqrt{\frac{8}{\pi}} \Lambda^{2} e^{-\Lambda^{4} s^{4} / 2}+\mathcal{O}\left(s^{6}\right)
$$

Lastly, we expand $\nabla_{\bar{z}^{\nu}} g_{r e t}^{\Lambda}$ in powers of $s$

$$
\nabla_{\bar{z}^{\mu}} g_{r e t}^{\Lambda}=\partial_{\bar{z}^{\mu}} g_{r e t}^{\Lambda}=\sigma_{\mu}\left(\frac{\partial \sigma}{\partial s}\right)^{-1} \frac{\partial g_{r e t}^{\Lambda}}{\partial s}=\left\{u_{\mu}(\tau)+\frac{s}{2} \frac{D u_{\mu}(\tau)}{d \tau}+\frac{s^{2}}{6} \frac{D^{2} u_{\mu}(\tau)}{d \tau^{2}}+\mathcal{O}\left(s^{3}\right)\right\} \frac{\partial g_{r e t}^{\Lambda}}{\partial s}
$$

Applying these results to (3.16) gives

$$
\begin{aligned}
&\left(m_{0}-\frac{e^{2}}{2} g_{(1)}(r)+e^{2} c_{(0)}(r)-e^{2} \int_{\tau_{i}}^{\tau} d \tau^{\prime} V\left(\bar{z}^{\alpha}, \bar{z}^{\alpha^{\prime}}\right)\right) \frac{D \bar{u}_{\mu}}{d \tau} \\
&=e w_{\mu}^{\nu}[\bar{z}] \phi_{\nu}^{\text {tail }}(\bar{z})+e^{2} \sum_{n=1}^{\infty}\left(g_{(n+1)}(r) u_{\alpha}^{(n+1)}[\bar{z}]+c_{(n)}(r) v_{\alpha}^{(n)}[\bar{z}]\right)
\end{aligned}
$$

with $r=\tau-\tau_{i}$ being the elapsed proper time since $\tau_{i}$.

The expressions for the $r$-dependent coefficients $\left(g_{(n)}\right.$ and $\left.c_{(n)}\right)$ and the trajectory-dependent vectors $\left(u_{\mu}^{(n)}\right.$ and $\left.v_{\mu}^{(n)}\right)$ can be found in Appendix B. However, only the relevant factors in the limit of very large $\Lambda$ will be given here

$$
\begin{array}{cc}
c_{(0)}(r)=-\frac{\Lambda}{2^{1 / 4} \sqrt{\pi}} \gamma\left(\frac{1}{4}, \frac{r^{4} \Lambda^{4}}{2}\right) & g_{(1)}(r)=-\Lambda \frac{2^{7 / 4}}{\sqrt{\pi}} \gamma\left(\frac{5}{4}, \frac{r^{4} \Lambda^{4}}{2}\right) \\
c_{(1)}(r)=\frac{1}{\sqrt{\pi}} \gamma\left(\frac{1}{2}, \frac{r^{4} \Lambda^{4}}{2}\right) & g_{(2)}(r)=\frac{2}{\sqrt{\pi}} \gamma\left(\frac{3}{2}, \frac{r^{4} \Lambda^{4}}{2}\right) \\
v_{\mu}^{(1)}[\bar{z}]=\frac{1}{6} w_{\mu}^{\nu}[\bar{z}] R_{\nu}{ }^{\alpha} \bar{u}_{\alpha} \\
u_{\mu}^{(1)}[\bar{z}]=\frac{1}{2} \bar{a}_{\mu} \quad u_{\mu}^{(2)}[\bar{z}]=\frac{1}{3} w_{\mu}^{\nu}[\bar{z}] \frac{D \bar{a}_{\nu}}{d \tau}
\end{array}
$$

where $\gamma(a, b)=\Gamma(a)-\Gamma(a, b)$ is the incomplete gamma function. The coefficients (4.11)-(4.12) vary over a timescale of $\sim \Lambda^{-1}$ after which they are effectively constant. 


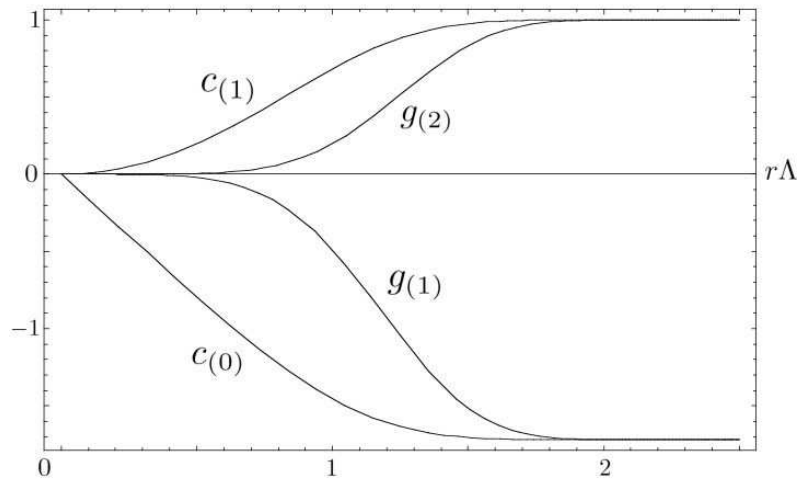

FIG. 1: Time-dependence of the coefficients appearing in 4.11-(4.12). The functions $c_{(0)}$ and $g_{(1)}$ have been divided through by $\Lambda$ so that they can be displayed along with $c_{(1)}$ and $g_{(2)}$.

Figure (11) shows the $r$-dependence of these functions. Note also that, at the initial time, all of these functions vanish. More will be said below concerning these properties and their implication for the validity of the quasilocal expansion.

\section{B. Scalar ALD Equation}

Most of the terms on the right side of (4.10) are irrelevant in the sense that they are inversely proportional to powers of $\Lambda$ so that for time-scales much longer than $\Lambda^{-1}$ these terms can be ignored. In this limit the effect of the high energy physics is ignorable and the effective particle dynamics is described by

$$
\begin{aligned}
m(\tau ; \bar{z}] & \frac{D \bar{u}_{\mu}}{d \tau} \\
= & F_{\mu}^{e x t}(\tau)+\frac{e^{2}}{3} g_{(2)}(r) w_{\mu}{ }^{\nu}[\bar{z}] \frac{D \bar{a}_{\nu}}{d \tau} \\
& +\frac{e^{2}}{6} c_{(1)}(r) w_{\mu}{ }^{\nu}[\bar{z}] R_{\nu \alpha}(\bar{z}) \bar{u}^{\alpha}+e w_{\mu}{ }^{\nu}[\bar{z}] \phi_{\nu}^{\text {tail }}(\bar{z}) \\
& +\mathcal{O}\left(\Lambda^{-1}\right)
\end{aligned}
$$

where the time-dependent and trajectory-dependent effective mass is

$$
\begin{aligned}
& m(\tau ; \bar{z}] \\
& =m_{0}-e^{2}\left(\frac{1}{2} g_{(1)}(r)-c_{(0)}(r)+\int_{\tau_{i}}^{\tau} d \tau^{\prime} V\left(\bar{z}^{\alpha}, \bar{z}^{\alpha^{\prime}}\right)\right)
\end{aligned}
$$

and an external force $F_{\mu}^{e x t}$ responsible for accelerating the charge has been included.

In the limit that $\Lambda \rightarrow \infty$, the terms involving $c_{(0)}(r)$ and $g_{(1)}(r)$ both diverge linearly with $\Lambda$ and the bare mass $m_{0}$ gets shifted by an infinite amount $\delta m=$ $e^{2}\left(g_{(1)} / 2-c_{(0)}\right)$. The mass is then renormalized to $m_{\text {ren }}=m_{0}-\delta m$, which is a constant with respect to $\tau$ in the infinite $\Lambda$ limit. If the regulator is finite but very large then the shift $\delta m$ is finite and large and the dressed mass has a very weak dependence on time (see Fig.(11)).

If we now make $\Lambda$ infinite and use the limiting values for $g_{(2)}$ and $c_{(1)}$

$$
\lim _{\Lambda \rightarrow \infty} g_{(2)}(r)=1=\lim _{\Lambda \rightarrow \infty} c_{(1)}(r)
$$

for a constant elapsed proper-time $r$ then the low-energy effective particle dynamics is

$$
m_{r e n}(\tau ; \bar{z}] \frac{D \bar{u}_{\mu}}{d \tau}=F_{\mu}^{e x t}(\tau)+f_{\mu}[\bar{z}]
$$

where the renormalized effective mass is

$$
m_{r e n}(\tau ; \bar{z}]=m_{r e n}-e^{2} \int_{\tau_{i}}^{\tau} d \tau^{\prime} V\left(\bar{z}^{\alpha}, \bar{z}^{\alpha^{\prime}}\right)
$$

and the self-force is

$$
f_{\mu}[\bar{z}]=w_{\mu}{ }^{\nu}[\bar{z}]\left(\frac{e^{2}}{3} \frac{D \bar{a}_{\nu}}{d \tau}+\frac{e^{2}}{6} R_{\nu \alpha}(\bar{z}) \bar{u}^{\alpha}+e \phi_{\nu}^{t a i l}(\bar{z})\right)
$$

Equations (4.18) through (4.20) are the main results of this section. The appearance of the tail term (3.17) implies that the radiation reaction is nonlocal because of its dependence on the past behavior of the classical worldline. This equation for the radiation reaction from scalar charges was first obtained by Quinn [1] based on earlier work of Quinn and Wald [4] and Mino, Sasaki and Tanaka [3] on gravitational radiation reaction.

Detweiler and Whiting [5] have obtained this result by decomposing the derivative of the retarded field $\phi_{\text {ret }}$ into a singular piece $\phi_{\mu}^{S}$, containing the diverging contribution that renormalizes the mass, and a regular piece $\phi_{\mu}^{R}$, which contributes to the self-force and is regular on the worldline. From the previous section we can construct these quantities within our regularization scheme to find that for $\Lambda \rightarrow \infty$

$$
\begin{aligned}
\phi_{\mu}^{S}= & -e \sqrt{\frac{8}{\pi}} \Lambda^{2} \bar{u}_{\mu}+e\left(\frac{1}{2} g_{(1)}(r)-c_{(0)}(r)\right) \frac{D \bar{u}_{\mu}}{d \tau} \\
\phi_{\mu}^{R}= & -e \frac{1-6 \xi_{R}}{12} R(\bar{z}) \bar{u}_{\mu}+\frac{e}{3} \frac{D \bar{a}_{\mu}}{d \tau}+\frac{e}{6} R_{\mu \alpha}(\bar{z}) \bar{u}^{\alpha} \\
& +\phi_{\mu}^{\text {tail }}(\bar{z})
\end{aligned}
$$

Notice that the first term of the singular part does not contribute to renormalizing any physical parameters (at the level of the equations of motion) since $\phi_{\mu}^{S}$ is projected onto a direction orthogonal to $\bar{u}_{\mu}$. Likewise, the first term of the regular part does not contribute to the self-force on the particle.

In a flat spacetime, the tail term vanishes since there is no curvature available to focus the radiation emitted in 
the past onto the particle at the present. Furthermore, (4.18) reduces to the ALD equation for a scalar field

$$
m_{r e n} \dot{\bar{u}}_{\mu}=F_{\mu}^{e x t}(\tau)+\frac{e^{2}}{3} w_{\mu}^{\nu}[\bar{z}] \ddot{\bar{u}}_{\nu}
$$

This equation was derived in the open quantum system formalism in [10].

\section{LINEARIZED FLUCTUATIONS AROUND THE CLASSICAL PARTICLE TRAJECTORY}

We now study the effects of the quantum field fluctuations (as classical stochastic forces) on the low energy dynamics of the particle. Instead of working directly with the equations for the linearized fluctuations (2.36), which contain the singular functional derivatives, we begin with (2.39) and assume that the retarded field has been regularized by the short-distance regulator $\Lambda$ in a quasi-local expansion.

\section{A. Stochastic ALD Equation}

For large finite values of $\Lambda$ (2.39) becomes the ALDLangevin equation

$$
m(\tau ; z] \frac{D u_{\mu}}{d \tau}=F_{\mu}^{e x t}(\tau)+f_{\mu}(z)+\eta_{\mu}(\tau ; z]
$$

where the (regulated) self-force is

$$
f_{\mu}[z]=w_{\mu}^{\nu}[z]\left(\frac{e^{2}}{3} g_{(2)}(r) \frac{D a_{\nu}}{d \tau}+\frac{e^{2}}{6} c_{(1)}(r) R_{\nu \alpha}(z) u^{\alpha}+e \phi_{\nu}^{\text {tail }}(z)\right)+\mathcal{O}\left(\Lambda^{(-1)}\right)
$$

Of course, one must remember that these expressions are only valid up to linear order in the fluctuations $\tilde{z}$ about the mean worldline $\bar{z}$ since higher orders correspond to quantum corrections that we have assumed to be negligible. Expanding (5.1) in orders of the fluctuations, the time-dependent mass and self-force are given by

$$
\begin{aligned}
m(\tau ; z] & =m(\tau ; \bar{z}]-e^{2} \int_{\tau_{i}}^{\tau} d \tau^{\prime} \tilde{z}^{\nu^{\prime}} \frac{\delta}{\delta \bar{z}^{\nu^{\prime}}} \int_{\tau_{i}}^{\tau} d \tau^{\prime \prime} V\left(\bar{z}^{\alpha}, \bar{z}^{\alpha^{\prime \prime}}\right)+\mathcal{O}\left(\tilde{z}^{2}\right) \\
f_{\mu}[z] & =f_{\mu}[\bar{z}]+\int d \tau^{\prime} \tilde{z}^{\nu^{\prime}} \frac{\delta}{\delta \bar{z}^{\nu^{\prime}}} f_{\mu}\left[\bar{z}^{\alpha}\right]+\mathcal{O}\left(\tilde{z}^{2}\right)
\end{aligned}
$$

Calculating the functional derivative in the mass equation gives

$$
m(\tau ; z]=m(\tau ; \bar{z}]-\frac{e}{2} \tilde{z}^{\nu} \phi_{\nu}^{t a i l}(\bar{z})-e^{2} \int_{\tau_{i}}^{\tau} d \tau^{\prime} \tilde{z}^{\nu^{\prime}} \nabla_{\bar{z}^{\prime}} V\left(\bar{z}^{\alpha}, \bar{z}^{\alpha^{\prime}}\right)+\mathcal{O}\left(\tilde{z}^{2}\right)
$$

where the factor of $1 / 2$ in the second term comes from evaluating a delta function on the upper boundary of the $\tau^{\prime}$ integral. Notice that the linear terms in $\tilde{z}$ vanish in flat space-time since $V$ is identically zero for a massless scalar field in $3+1$ dimensions.

Simplifying the self-force fluctuations is slightly more involved. The calculation amounts to performing the variational derivative on $f_{\mu}$ but keeping in mind to expand out the covariant derivatives, which depend on the classical worldline coordinates. The result is

$$
f_{\mu}[z]=f_{\mu}[\bar{z}]-\kappa_{\mu \alpha}[\bar{z}] \tilde{z}^{\alpha}-\gamma_{\mu \alpha}[\bar{z}] \dot{\tilde{z}}^{\alpha}-m_{\mu \alpha}[\bar{z}] \ddot{\tilde{z}}^{\alpha}+r_{\mu \alpha}[\bar{z}] \dddot{\tilde{z}}^{\alpha}+\mathcal{O}\left(\Lambda^{-1}\right)
$$

The time- and trajectory-dependent coefficients are complicated expressions that are not particularly illuminating. These are recorded in Appendix C.

Combining the linearized mass and self-force into (5.1) and using the fact that $\bar{z}$ satisfies the mean equations of motion (2.23) results in

$$
M_{\mu \alpha}[\bar{z}] \ddot{\tilde{z}}^{\alpha}+\Gamma_{\mu \alpha}[\bar{z}] \dot{\tilde{z}}^{\alpha}+K_{\mu \alpha}[\bar{z}] \tilde{z}^{\alpha}-e^{2} \bar{a}_{\mu} \int_{\tau_{i}}^{\tau} d \tau^{\prime} \tilde{z}^{\nu^{\prime}} \nabla_{\bar{z}^{\prime}} V\left(\bar{z}^{\alpha}, \bar{z}^{\alpha^{\prime}}\right)=r_{\mu \alpha}[\bar{z}] \dddot{\tilde{z}}^{\alpha}+\eta_{\mu}[\bar{z}]+\mathcal{O}\left(\Lambda^{-1}\right)
$$

where

and

$$
\begin{aligned}
K_{\mu \alpha}[\bar{z}] & =\kappa_{\mu \alpha}[\bar{z}]-m(\tau ; \bar{z}] \partial_{\bar{z}^{\alpha}} \Gamma_{\mu \gamma}^{\beta} \bar{u}_{\beta} \bar{u}^{\gamma}-\frac{e}{2} \bar{a}_{\mu} \phi_{\alpha}^{\text {tail }}(\bar{z}) \\
M_{\mu \alpha}[\bar{z}] & =g_{\mu \alpha} m(\tau ; \bar{z}]+m_{\mu \alpha}[\bar{z}]
\end{aligned}
$$

$$
\Gamma_{\mu \alpha}[\bar{z}]=\gamma_{\mu \alpha}[\bar{z}]-2 m(\tau ; \bar{z}] \Gamma_{\mu(\alpha}^{\beta} \bar{u}_{\beta)}
$$


The dynamical equation (5.7) for the fluctuations about the classical particle trajectory is the main result of this section. This is a linear integro-differential equation for $\tilde{z}$ with a third derivative term and contains time-dependent coefficients that depend on the non-Markovian behavior of the mean trajectory. Furthermore, because of the integration over past times the last term on the left side depends on the history of the fluctuations. Notice that this term vanishes in a flat background so that the fluctuations then obey a third-order differential equation, which is Markovian in the sense that given a mean trajectory $\bar{z}^{\mu}$ the fluctuations do not depend on their own past history. So, given a solution to the mean equation of motion for the classical worldline one could, in principle, solve for the fluctuations induced by the quantum field fluctuations.

The notation in (5.7) has been chosen suggestively since the left side resembles a damped simple harmonic oscillator with time-dependent mass, damping factor, and time- and history-dependent spring constant. Notice also that the "effective mass" $M_{\mu \alpha}[\bar{z}]$ is not diagonal implying that the inertia of the fluctuations behaves differently in different directions. This feature is exhibited in all of the other coefficients $\left(\Gamma_{\mu \alpha}, K_{\mu \alpha}\right.$ and $\left.r_{\mu \alpha}\right)$ and suggests that the fluctuations of the trajectory in one direction are linked with the fluctuations in the other space-time directions. Also, from the expression for the radiation reaction on the fluctuations $r_{\mu \alpha}[\bar{z}]$ it should be noted that this is explicitly independent of the background curvature and effectively projects $\ddot{\tilde{z}}_{\nu}$ onto a direction orthogonal to the mean 4-velocity $\bar{u}_{\nu}$.

If the stochastic term $\eta_{\mu}$ is ignored then (5.7) describes the evolution of small perturbations away from the semiclassical trajectory and so is useful for studying the linear response of the trajectory to small perturbations away from the mean worldline. In other words, setting $\eta_{\mu}$ to zero (5.7) gives the linearization of the ALD-Langevin equation around the semiclassical worldline $\bar{z}$. Generalizing this to the self-force due to linearized metric perturbations could be useful for investigating the stability of numerical calculations of the inspiral of a small mass black hole into a large mass black hole, for example [30].

\section{B. Memory and Secular Effects}

A particularly interesting feature related to this is the effect of the non-Markovian term appearing in (5.7)

$$
-e^{2} \bar{a}_{\mu} \int_{\tau_{i}}^{\tau} d \tau^{\prime} \tilde{z}^{\nu^{\prime}} \nabla_{\bar{z}^{\nu^{\prime}}} V\left(\bar{z}^{\alpha}, \bar{z}^{\alpha^{\prime}}\right)
$$

If the fluctuations $\tilde{z}$ grow then the linearization may not be valid for all times and so one should then include quantum corrections to our stochastic semi-classical equations if the environment is a quantum field as is assumed here. The growth of the fluctuations could be significantly influenced by the past behavior of $\nabla_{\nu} V$ and the history of the fluctuations. The behavior of this term on the fluctuations is not known yet because of the difficulty in solving integrodifferential equations and in computing $V$ for many spacetimes. However, a study in de Sitter space (where $V$ is known 6, 19]) may provide a simple arena for understanding the role of the non-Markovian term and the nature of the solutions to (5.7).

Aside from these technical considerations, these equations are applicable for any type of noise on the particle trajectories. In many cases, the source of the noise acting on the system of interest may not be known and is put in by hand in a phenomenological description of the particle dynamics via the stochastic equation

$$
m(\tau ; z] \frac{D u_{\mu}}{d \tau}=F_{\mu}^{e x t}(\tau)+f_{\mu}(z)+\eta_{\mu}^{+}
$$

where the superscript $(+)$ is to remind us that this term was added in by hand, as opposed to being derived, like our treatment of $\eta_{\mu}[\bar{z}]$. This stochastic force could have a classical origin (e.g. high-temperature thermal fluctuations of surrounding gas) or it could have no known single identifiable origin. Furthermore, since the noise $\eta_{\mu}^{+}$is not derived from an initially closed system it is likely to be inconsistent with the dynamics of the trajectory. (See, e.g., 15]) In any case, one would also have to specify the noise correlator $\left\langle\eta_{\mu}^{+}(\tau) \eta_{\mu^{\prime}}^{+}\left(\tau^{\prime}\right)\right\rangle_{\eta^{+}}$as it suits the model.

With this proviso (no guarantee for consistency) the analysis of this section carries over. Given any kind of noise the fluctuations around the mean trajectory of the particle moving through its own (classical) field subjected to the self-force from radiation reaction is given by (5.7), but with $\eta_{\mu}[\bar{z}]$ replaced by $\eta_{\mu}^{+}$

$$
\begin{aligned}
& M_{\mu \alpha}[\bar{z}] \ddot{z}^{\alpha}+\Gamma_{\mu \alpha}[\bar{z}] \dot{\tilde{z}}^{\alpha}+K_{\mu \alpha}[\bar{z}] \tilde{z}^{\alpha} \\
& -e^{2} \bar{a}_{\mu} \int_{\tau_{i}}^{\tau} d \tau^{\prime} \tilde{z}^{\nu^{\prime}} \nabla_{\bar{z}^{\nu^{\prime}}} V\left(\bar{z}^{\alpha}, \bar{z}^{\alpha^{\prime}}\right) \\
& =r_{\mu \alpha}[\bar{z}] \dddot{\tilde{z}}^{\alpha}+\eta_{\mu}^{+}+\mathcal{O}\left(\Lambda^{-1}\right)
\end{aligned}
$$

Since in this discussion we don't need to worry about quantum corrections from higher order loops in the effective action we could go beyond the linear order in the fluctuations of the particle trajectory and expand the solutions to (5.11) in powers of the coupling constant $e$ ( denoted in the subscript) $z=z_{0}+z_{1}+z_{2}+\ldots$.. Assuming that $\eta_{\mu}^{+}$is $\mathcal{O}(e)$ and depends on $z$ and recalling that $f_{\mu}$ is quadratic in the coupling we find a non-local and causal contribution to the total force on the particle coming from the fluctuations of the stochastic force (see Appendix D for details)

$F_{\mu}^{d r i f t}=\int d \tau^{\prime} d \tau^{\prime \prime} F_{\mu}^{\rho \sigma}\left(\tau, \tau^{\prime}, \tau^{\prime \prime}\right)\left\langle\eta_{\rho}^{+}\left(z_{0}^{\alpha^{\prime}}\right) \eta_{\sigma}^{+}\left(z_{0}^{\alpha^{\prime \prime}}\right)\right\rangle_{\eta^{+}}$

which is of the same order as the self-force. It seems that the stochastic noise would cause the particle to drift off from the background trajectory $z_{(0)}$ determined by the external force. The deviation could build up over 
time as indicated by the integral above. This may have interesting consequences for astrophysical sources with some stochastic behavior described by a classical noise. If such physical situations exist, this noise-induced drift may give rise to a secular effect which could alter the waveform templates (of events expected to be seen by gravitational interferometers like LIGO and LISA) calculated without including such stochastic secular effects.

A similar expression to (5.13) can be derived in our open quantum system framework by writing the effective action to cubic order in the fluctuations. Doing this reveals a term like (5.13) but this requires a much more careful analysis that goes beyond the stochastic semiclassical approximation adopted here.

\section{DISCUSSIONS}

\section{A. The Quantum Regime and the Validity of the Quasi-Local Expansion and Order-Reduction}

The previous sections focused on the Feynman-Vernon formalism and various approximations to obtain the lowenergy effective dynamics of the particle, both for its semiclassical (mean) and stochastic semi-classical (mean and stochastic fluctuations) motion. Here, the domain of validity of the quasi-local expansion and this semiclassical treatment will be explored and compared with the relevant scales for weak and strong radiation damping.

In the effective field theory paradigm a regulator $\Lambda$ is introduced for controlling the ultraviolet divergences appearing in the direct part of the retarded Green's function such that $\Lambda^{2} \sigma \gg 1$ with $\sigma$ small and approaching zero. After expanding $\sigma$ near the coincidence limit the timescale of the quasi-local expansion $\Delta \tau=s$ is governed by

$$
\Delta \tau \gg \Lambda^{-1}
$$

Recall that for times larger than $\sim \Lambda^{-1}$ the timedependent coefficients in (4.15) and (5.1) rapidly approach constant values (see Fig [1). But there are other scales to consider. We have been working at tree-level in both the particle and the field so that $\Delta \tau$ must be much larger than the scale for 1-loop field and 1-loop particle corrections. This implies that the time interval $\Delta \tau$ should be much longer than the time-scale for creating pairs

$$
\Delta \tau \gg \frac{\hbar}{m}=\lambda_{C}
$$

where $\lambda_{C}$ is the Compton wavelength associated with the scalar particle. The dynamics (4.15) and (5.1) are therefore valid using this framework provided that

$$
\Delta \tau \gg \lambda_{C} \gg \Lambda^{-1}
$$

Nevertheless, the presence of the third $\tau$-derivative in (4.15), (5.1) and (5.7) requires the specification of the initial position, velocity, and acceleration to obtain unique solutions. This is problematic, since for a vanishing external force $F_{\mu}^{e x t}$ one still requires an initial acceleration to solve the equations. But, if there is no force accelerating the charge then what causes the particle to accelerate? Furthermore, the particle may experience unbounded acceleration so that its kinetic energy increases with time to infinity. Since scalar dynamics is an energyconserving theory where then could this energy arise? These problems are related to the infinite self-energy of a (classical) charged point particle.

In the classical theory of scalar fields and particles these issues can be resolved if one instead gives the particle a finite size $r_{0}$ for which the self-energy is roughly $e^{2} / r_{0}$ 31]. If this energy composes its rest mass then

$$
r_{0} \sim \frac{e^{2}}{m}
$$

and represents the "size" of the particle. In [31], an approximation, amounting to an asymptotic expansion in powers of $r_{0}$, called the Landau approximation (also known as order-reduction), is employed to obtain solutions that require only an initial position and velocity and are also free from run-away solutions. The Landau approximation converts the ALD equation (of third order) to the so-called Landau-Lifshitz equation (of second order). We will use these names to distinguish between these equations.

In order-reduction, the lowest order solution is found by simply ignoring the self-force so that the radiation damping is assumed weak. The time-scale of the dynamics is then determined mostly by the external force so that if $F_{\mu}^{e x t}$ varies on a scale $\lambda_{\text {ext }}$ then $\Delta \tau \sim \lambda_{\text {ext }}$. In curved spacetimes the self-force will be weak if $r_{0} \ll \Delta \tau$ and the length scale associated with the spacetime curvature $\lambda_{R}$ is large (i.e. small curvature) so that $\lambda_{R} \gg r_{0}$. It then follows that for weak damping in the semi-classical domain that the quasi-local expansion and the Landau approximation are valid provided that

$$
\Delta \tau \sim \lambda_{\text {ext }} \gg r_{0}, \lambda_{C} \gg \Lambda^{-1} \text { and } \lambda_{R} \gg r_{0}
$$

Recently, in the context of plasma physics, 29] has investigated the validity of the Landau approximation for the classical ALD equation by numerically integrating the Landau-Lifshitz equation forward in time and, using the final position, velocity, and acceleration from that, integrating the ALD equation backward in time. If the initial position and velocity of the particle differ significantly from the backward-evolved solution of the ALD equation at the initial time then one can assume the Landau approximation has broken down. Koga does this for a counter-propagating electron and ultraintense laser beam (intensity $\sim 10^{22} \mathrm{~W} / \mathrm{cm}^{2}$ ). He finds that the Landau approximation is valid so long as the laser wavelength $\lambda_{0}$ is greater than the Compton wavelength. For 
$\lambda_{0}$ much smaller than $\lambda_{C}$, he finds disagreement between the solutions of the Landau-Lifshitz and ALD equations. However, these equations cannot be fully trusted since quantum effects should become important. In this domain, while we cannot directly apply our results to this problem we can use the closely related closed-time-path (CTP) formalism to incorporate the effects of the quantum loop corrections to the (quantum) particle dynamics.

\section{B. Decoherence}

This brings us to an important issue, namely, how strongly must the particle worldline decohere in order for a stochastic semi-classical treatment to be applicable? A measure of the decoherence of a system is the decoherence functional [32] $D\left[\alpha(z), \alpha\left(z^{\prime}\right)\right]$ where $\alpha(z)$ specifies a particular coarse-grained history of the worldline. If we take the history to be $\alpha(z)=z$ then the decoherence functional is

$$
\begin{aligned}
D\left[\alpha(z), \alpha\left(z^{\prime}\right)\right] & =D\left[z, z^{\prime}\right] \\
& =\rho_{S}\left(z_{i}, z_{i}^{\prime}\right) e^{\frac{i}{\hbar} S_{C G E A}\left[z, z^{\prime}\right]}
\end{aligned}
$$

and is closely related to the CTP effective action [33]. In fact, the norm of $D$ is proportional to the norm of the influence functional. The system is said to decohere if $|D|$ approaches zero. We showed earlier that the CGEA is proportional to $z^{-}$and so there is minimal decoherence for two nearby histories. This implies that coarse-graining the quantum field must be complemented by an additional coarse-graining of the worldline in order for the particle to be sufficiently decohered. If the mechanism for decoherence is efficient then this additional coarse-graining should be minimal and the stochastic semi-classical description that we have used in this paper is applicable. For our problem, recall that the CGEA contains an imaginiary part that is proportional to $j^{-} \cdot G_{H} \cdot j^{-}$so that the norm of $D$ is

$$
\left|D\left[z, z^{\prime}\right]\right|=e^{-\frac{1}{4 \hbar} j^{-} \cdot\left(16 \pi^{2} G_{H}\right) \cdot j^{-}}
$$

showing that the quantum field fluctuations in the environment is the mechanism for decoherence. It is therefore necessary to understand the behavior of the Hadamard function to show that there exists a well-defined stochastic semi-classical regime for the particle. This requires knowing the field modes on the background space-time, which is a significant problem in its own right. For simple scenarios the decoherence functional can be calculated and the decoherence time approximated. But, in general, one needs to determine if decoherence is fast enough on a case by case basis.

\section{Problems with putting noise in by hand}

Towards the end of Sec. 5 we replaced the classical stochastic manifestation of the quantum fluctuations by a noise source $\eta_{\mu}^{+}$that has some specified noise correlator. Aside from the issue about keeping only to tree-level in the particle and field variables, how is using $\eta_{\mu}^{+}$different from all of the sophisticated machinery used in the previous sections?

Unlike in our approach, inserting a source of noise by hand implies that it can have any correlator one wants. The stochastic two-point function of $\eta_{\mu}^{+}$

$$
\left\langle\left\{\eta_{\mu}^{+}(\tau), \eta_{\nu}^{+}\left(\tau^{\prime}\right)\right\}\right\rangle_{\eta^{+}}=N_{\mu \nu}\left(\tau, \tau^{\prime}\right)
$$

otherwise known as the noise kernel, can be chosen at will. Some physical reasoning, for instance, a high temperature environment, might suggest that $N_{\mu \nu}\left(\tau, \tau^{\prime}\right) \sim$ $\delta\left(\tau-\tau^{\prime}\right)$, i.e. white noise. While white noise certainly simplifies the calculations, there is little justification for simply stating the form of the noise kernel and expecting the dynamics of the system to be consistent with that source. Using the Feynman-Vernon formalism, coarsegraining over the quantum environment naturally gives rise to the noise kernel appropriate for the stochastic force fluctuations

$$
\begin{aligned}
& \left\langle\left\{\eta_{\mu}\left[\bar{z}^{\alpha}\right], \eta_{\nu}\left[\bar{z}^{\alpha^{\prime}}\right]\right\}\right\rangle_{\xi} \\
& =\hbar e^{2} \vec{w}_{(\mu}\left[\bar{z}^{\alpha}\right] \vec{w}_{\nu)}\left[\bar{z}^{\alpha^{\prime}}\right] G_{H}\left(\bar{z}^{\alpha}, \bar{z}^{\alpha^{\prime}}\right)
\end{aligned}
$$

This coarse-graining ensures that the dynamics of the system evolves consistently with that of the environment. This is an important statement that cannot be overstated because it is this feature which gives rise to consistent fluctuation-dissipation relations (FDR). Inserting noise by hand most likely violates such a relation.

While FDR's won't be discussed here in great detail, 7] have studied the FDR's for $n$ particles interacting with a quantum field for certain trajectories (e.g. uniformly accelerated). They have shown the existence and self-consistency of these relations and further introduce correlation-propagation relations relating the correlations of particles to each other through the (causal) propagation of the quantum field. These correlationpropagation relations can only be formed if the particles interact directly through causal influences. The open quantum system viewpoint, therefore, shows the interrelated influences of the system and environment through these types of self-consistent relations. This is an important feature lacking in a model that has noise put in by hand.

Another problem with adding noise in by hand is that the noise can be a significant contribution to the quantum two-point function of the system if one is interested in the quantum particle behavior. So, having the correct form for the noise kernel is important for getting the correct expression for the quantum two-point functions.

It has been shown in [18], using the CTP generating functional for quantum correlation functions of the system variables, for quantum Brownian motion as well as for stochastic semi-classical gravity (see next section), that certain quantum two-point functions are related to 
stochastic two-point functions. As an example, the symmetrized quantum two-point function of a Brownian particle's position can be written as

$$
\frac{1}{2}\left\langle\left\{\hat{x}(t), \hat{x}\left(t^{\prime}\right)\right\}\right\rangle=\left\langle\left\langle X(t) X\left(t^{\prime}\right)\right\rangle_{\xi}\right\rangle_{X_{i}, p_{i}}
$$

where $X$ is a solution of the QBM Langevin equation $L \cdot X=\xi$ for the appropriate linear operator $L\left(t, t^{\prime}\right)$, and
$\langle\ldots\rangle_{X_{i}, p_{i}}$ denotes the average over all possible initial positions and momenta with respect to the reduced Wigner function for the initial state of the particle. The solutions of the QBM Langevin equation consist of a homogeneous part, containing all the information about the initial conditions of the system, and a term describing effects due to the interactions between the particle and oscillators.

$$
X(t)=X_{0}(t)+\int d t^{\prime} G_{r e t}\left(t, t^{\prime}\right) \xi\left(t^{\prime}\right)
$$

The symmetrized quantum two-point function becomes

$$
\frac{1}{2}\left\langle\left\{\hat{x}(t), \hat{x}\left(t^{\prime}\right)\right\}\right\rangle=\left\langle X_{0}(t) X_{0}\left(t^{\prime}\right)\right\rangle_{X_{i}, p_{i}}+\int d t_{1} \int d t_{2} G_{r e t}\left(t, t_{1}\right) N\left(t_{1}, t_{2}\right) G_{r e t}\left(t_{2}, t^{\prime}\right)
$$

where $N=\left\langle\xi\left(t_{1}\right) \xi\left(t_{2}\right)\right\rangle_{\xi}$ is the noise kernel, given by the stochastic correlator of $\xi$. The first term involving the homogeneous solution of the Langevin equation represents the dispersion in the initial conditions and is called the intrinsic fluctuations. The second term involving the noise kernel, and hence the quantum fluctuations of the environment, represents the correlations with the environment through the stochastic fluctuations and is called the induced fluctuations. It turns out that if the homogeneous solution $X_{0}$ decays exponentially fast then for late times, at least times larger than the decay time, the quantum two-point function is determined entirely by the noise kernel, that is, the induced fluctuations. And so, in this sense, all of the information about the quantum correlations of the system degrees of freedom is encoded in the stochastic correlations. This shows another drawback to putting noise in by hand. The induced fluctuations contain information about the quantum fluctuations of the system variables. But this will not be true with some arbitrary noise kernel chosen at will.

\section{Similarities with Stochastic Semiclassical Gravity}

The features of the particle dynamics seen in the above discussions are typical of nonequilibrium open quantum systems. History-dependent behavior is present in the equations of motion for the system and if a renormalization procedure is required it is usually a time-dependent prescription, as seen earlier with the renormalized mass $m_{\text {ren }}(\tau)$. Furthermore, the noise correlator (6.10) is generically non-local in time and is determined by the quantum fluctuations of the environment variables. This formalism does not allow for arbitrary noise kernels since this would destroy the self-consistency between the sys- tem and environment evolution. A particular example that contains these features is stochastic semiclassical gravity, which we will briefly describe and compare with below.

Stochastic semiclassical gravity (SSG) is a selfconsistent theory of the stochastic dynamics of a classical spacetime containing quantum matter fields. SSG goes beyond semiclassical gravity, for which the geometry is driven by the expectation of the (renormalized) stress tensor, in that the quantum field fluctuations also contribute to the spacetime dynamics through a classical stochastic source. The spacetime is therefore driven by both the quantum expectation value of the renormalized stress tensor and a classical stochastic stress-tensor-like object, $\xi_{a b}$. For an introduction and review of this subject see [34] and 35] for a discussion of the domain of validity of SSG.

As an open quantum system, the quantum field fluctuations are coarse-grained using the CTP formalism of Schwinger and Keldysh (SK) to study the self-consistent evolution of the (classical) geometry. The quantum fluctuations manifest themselves as stochastic noise thereby imparting a stochastic nature to the spacetime. The resulting Einstein-Langevin equation for the linearized metric perturubations $h_{a b}$ is

$$
G_{a b}^{(1)}[g+h]=\kappa\left\langle\hat{T}_{a b}^{(1)}[g+h]\right\rangle_{r e n}+\kappa \xi_{a b}[g]
$$

The superscript ${ }^{(1)}$ denotes that those quantities contain all terms to first order in the metric fluctuations $h_{a b}$. It should be noted that the finite parts of the counterterms needed to cancel the divergences coming from the stress tensor expectation value have been absorbed into the definition of $\left\langle\hat{T}_{a b}^{(1)}\right\rangle_{\text {ren }}$. The renormalized stress tensor expectation value (evaluated in a Gaussian state) contains an integration over the past history of the metric fluctuations and so the dynamics is generally non- 
Markovian. This is like what is seen in the ALD-Langevin equation (5.1) where the tail term $\phi_{\nu}^{\text {tail }}(z)$ is analogous to the expectation value of the renormalized stress tensor in (6.14). The (covariantly conserved) stochastic source tensor $\xi_{a b}$ has zero mean and its correlator is given in terms of the Hadamard function of the stress tensor fluctuations $\hat{t}_{a b}=\hat{T}_{a b}-\left\langle\hat{T}_{a b}\right\rangle$

$$
\left\langle\left\{\xi_{a b}(x ; g], \xi_{c d}\left(x^{\prime} ; g\right]\right\}\right\rangle_{\xi}=\hbar\left\langle\left\{\hat{t}_{a b}(x ; g], \hat{t}_{c d}\left(x^{\prime} ; g\right]\right\}\right\rangle
$$

The correlator of the stress tensor fluctuations on the right side does not vanish on a spatial hypersurface. This reflects the fact that the quantum field correlations are themselves non-local. Compare this with the correlator in (2.31) which is also non-local.

SSG also suffers from runaway solutions since the finite contributions to the counterterms needed to cancel the divergences appearing from the expectation value of the stress tensor are quadratic in the curvature. This makes SSG a theory with derivatives higher than two, similar to the ALD type equations derived above, which were of third order in the $\tau$ derivatives. A version of the Landau approximation can be used to reduce the order of the Einstein-Langevin equation to two thereby yielding well-behaved solutions free of the pathologies typical of higher-order derivative theories. Of course, one needs to be careful to use the Landau approximation at scales that are consistent with the derivation of the EinsteinLangevin equation.

Finally, the symmetrized quantum two-point functions of the metric fluctuations $h_{a b}$ can be written in terms of intrinsic fluctuations, representing the dispersion in the initial conditions, and induced fluctuations, encoding the information about the fluctuations of the quantum matter 35. Just like with the particle motion, one cannot simply use any noise kernel for modeling stochastic metric fluctuations. One needs to do a careful analysis that ensures the self-consistency of the metric and quantum matter dynamics and the existence of fluctuationdissipation relations.

\section{SUMMARY}

In this paper we have derived the scalar ALD equation for the quantum expectation value of the worldline for a scalar charged point particle interacting with its own quantum field as it moves in a curved spacetime. Our equation (4.18) for the low-energy effective particle dynamics agrees with the results obtained earlier by $1,[5,6,19$. If the quantum fluctuations in the field strongly decohere the worldline then we can ignore the particle's quantum fluctuations and obtain the semiclassical motion (4.18). Invoking an effective field theory point of view, the singular behavior of the field's retarded Green's function can be regulated. For sufficiently short times $\Delta \tau=s$ but still long enough $\Delta \tau \gg \Lambda^{-1}$ compared with the inverse cutoff frequency $\Lambda$ a quasi-local expansion can be used to obtain the contributions relevant to the self-force and those that are irrelevant in the infinite $\Lambda$ limit. This renormalizes the mass of the particle and shows explicitly the appearance of the expected $D a_{\mu} / d \tau$ term that is characteristic of radiation reaction.

While the time-dependent coefficients (4.11)-(4.12) appearing in (4.15) seem to suggest that only the initial position and velocity of the particle are needed, and hence that a resolution of the problems of pre-acceleration and run-away solutions has been reached, one should keep in mind that the quasi-local approximation breaks down for short time intervals, which includes the instant at the initial time. A more careful analysis would need to include a more physical initial state than the factorized one used here.

Fluctuations in the quantum field is expected to affect the particle's motion causing it to fluctuate by an amount $\tilde{z}$ around the mean trajectory $\bar{z}$ given by solutions to the semi-classical equation 4.15). We derived such a stochastic force $\eta_{\mu}[\bar{z}]$ with correlators from the fluctuations of the quantum field and a scalar ALD-Langevin equation (5.1). The dynamics of $\tilde{z}$ (5.7) contains a nonMarkovian contribution through the past history of the particle fluctuations. Depending on the behavior of this term the fluctuations might grow to be large indicating a breakdown of the linear approximation. In that case it requires the inclusion of quantum corrections in order to follow the nonlinear evolution of $\tilde{z}$. On the other hand, ignoring the noise altogether in (5.7), one can test the stability of numerical simulations in inspiral studies, for example [30].

Instead of the noise $\eta_{\mu}$ derived here from fluctuations of quantum fields one can replace it with some other classical noise $\eta_{\mu}^{+}$suitably chosen to model some stochastic source in a phenomenological description. We can still use (5.7) to study the effect of such noises on the particle trajectory fluctuations. However, since the origin for the noise is no longer due to a quantum field we need not worry about keeping up to linear order in $\tilde{z}$ in the ALD-Langevin equation. Instead, expanding the solution for small coupling constant $e$ and taking the stochastic expectation value shows that there is, in general, a non-vanishing force (5.13) coming completely from the correlations of the stochastic force. Along with the selfforce, this noise-induced term would cause the particle to drift off of its background trajectory determined by the external force (or off of its geodesic motion if $F_{\mu}^{e x t}$ vanishes). We hope to explore the consequences of this noiseinduced drift in an astrophysical setting and find observable effects on the waveforms of the radiation emitted by the particle and detected by gravitational interferometers like LIGO and LISA.

In Paper II we will apply the same techniques here to study the self-forces and the stochastic semi-classical motions of electric charges coupled to an electromagnetic field and of small black holes coupled to the background spacetime of a massive black hole, respectively. We hope 
to apply these results to more physical situations such as the motion of charges in strong external fields and gravitational radiation reaction.

\section{Acknowledgments}

CRG thanks Phil Johnson for help in learning the worldline influence functional approach approach and Albert Roura for suggestions in simplifying some derivations in Appendix A. We both thank Phil Johnson and Albert Roura for interesting discussions on general issues of radiation reaction. This work is supported in part by NSF grant PHY03-00710.

\section{APPENDIX A: GEODESIC COORDINATES AND THE QUASI-LOCAL EXPANSION}

In this Appendix we derive the quasi-local expansion of $\sigma^{\alpha}$ in (4.7) and some of the relations appearing in Section IV for computing the quasi-local expansion of the regulated direct part of the retarded Green's function.

We begin by invoking the so-called geodesic coordinates along and near the worldline [31, 36]. These coordinates will be denoted with a hat. In geodesic coordinates the metric tensor and the connection coefficients are constructed to vanish along the entirety of the worldline

$$
\left.\hat{g}_{\mu \nu}\right|_{\gamma}=\left.0 \quad \hat{\Gamma}_{\mu \nu}^{\lambda}\right|_{\gamma}=0
$$

so that a vector field on the worldline behaves like a vector field in flat spacetime. The displacement from $A$ to $B$ in Fig(2) can be written as

$$
\Delta \hat{z}^{\alpha} \stackrel{\text { def }}{=} \hat{z}^{\alpha}-\hat{z}^{\alpha^{\prime}}
$$

Using a Taylor series to express $\hat{z}^{\alpha^{\prime}}$ in terms of $\hat{z}^{\alpha}$ and its derivatives results in

$$
\begin{aligned}
\Delta \hat{z}^{\alpha} & =-s \hat{u}^{\alpha}-\frac{s^{2}}{2 !} \frac{d \hat{u}^{\alpha}}{d \tau}-\frac{s^{3}}{6 !} \frac{d^{2} \hat{u}^{\alpha}}{d \tau^{2}}-\ldots \\
& =-s \hat{u}^{\alpha}-\frac{s^{2}}{2 !} \frac{D \hat{u}^{\alpha}}{d \tau}-\frac{s^{3}}{6 !} \frac{D^{2} \hat{u}^{\alpha}}{d \tau^{2}}-\ldots
\end{aligned}
$$

where, in the last line, we have used the fact that the components of the connection vanish in this coordinate system.

Flatness along the worldline implies that the tangent spaces at each point on $\gamma$ can be identified with each other so that tensor manipulations along $\gamma$ can be done in a single tangent space. This implies that $\Delta \hat{z}^{\alpha}$ is a vector in the tangent space that we can interpret as a displacement vector at $B$. Transforming back to the original coordinates gives

$$
\begin{aligned}
\Delta z^{\alpha} & =\frac{\partial x^{\alpha}}{\partial \hat{x}^{\beta}} \Delta \hat{z}^{\beta} \\
& =-s u^{\alpha}-\frac{s^{2}}{2 !} a^{\alpha}-\frac{s^{3}}{6 !} \frac{D a^{\alpha}}{d \tau}-\ldots
\end{aligned}
$$

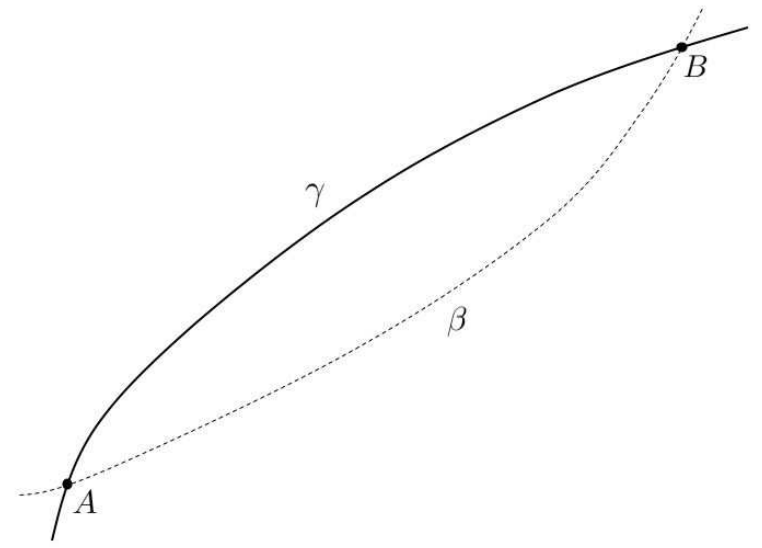

FIG. 2: For two points on a worldline $\gamma$ one can construct a unique time-like geodesic $\beta$ connecting them.

Using geodesic coordinates appropriate for the geodesic connecting $A$ and $B$ (denoted with a bar instead of a hat) one finds that

$$
\bar{\sigma}^{\alpha}=\left(\lambda-\lambda^{\prime}\right) \bar{t}^{\alpha}=\bar{y}^{\alpha}-\bar{y}^{\alpha^{\prime}}
$$

which represents the coordinate difference between $A$ and $B$ in the tangent space at $B$. Here we take $\bar{t}^{\alpha}$ to be the tangent vector to the geodesic $\beta$ connecting $A$ and $B$ with affine parameter $\lambda^{\prime \prime}$ and with coordinates $y$ such that $\left.y\right|_{A}=y\left(\lambda^{\prime}\right)$ and $\left.y\right|_{B}=y(\lambda)$. In the original coordinates

$$
\sigma^{\alpha}=\frac{\partial x^{\alpha}}{\partial \bar{x}^{\beta}} \bar{\sigma}^{\beta}
$$

Since $\Delta \hat{z}^{\alpha}$ and $\bar{\sigma}^{\alpha}$ both represent the (coordinate) difference between $A$ and $B$ in the same tangent space then they are related by the coordinate transformation from the hat to the bar coordinates

$$
\bar{\sigma}^{\beta}=\frac{\partial \bar{x}^{\beta}}{\partial \hat{x}^{\gamma}} \Delta \hat{z}^{\gamma}
$$

This immediately implies that

$$
\begin{aligned}
\sigma^{\alpha}\left(z^{\alpha}, z^{\alpha^{\prime}}\right) & =-s u^{\alpha}-\frac{s^{2}}{2 !} a^{\alpha}-\frac{s^{3}}{6 !} \frac{D a^{\alpha}}{d \tau}-\ldots \\
& =-\sum_{n=1}^{\infty} \frac{s^{n}}{n !} \frac{D^{n}}{d \tau^{n}} u^{\alpha}(\tau)
\end{aligned}
$$

There is an interesting interpretation one may attach to this result. Pick a point $x^{\prime}$ in the normal convex neighborhood of $x$ in some spacetime. These points are connected by a unique geodesic described by $\sigma^{\alpha}$. If these two points intersect any worldline describing the motion of some particle then in terms of the particle's velocity and its derivatives $\sigma^{\alpha}=\Delta z^{\alpha}$. This equality therefore describes a (many-to-one) mapping between particle motions and geodesics. 
From the identity $2 \sigma=\sigma_{\alpha} \sigma^{\alpha}$ it is possible to show that

$$
\sigma=-\frac{s^{2}}{2}-\frac{s^{4}}{24}+\mathcal{O}\left(s^{5}\right)
$$

This also provides a relation between the parametrization of the geodesic and the proper time of the worldline through

$$
\left(\lambda-\lambda^{\prime}\right)^{2}=s^{2}+\frac{s^{4}}{12}+\mathcal{O}\left(s^{5}\right)
$$

since $2 \sigma=-\left(\lambda-\lambda^{\prime}\right)^{2}$ is the amount of elapsed proper time along the geodesic.

Using these results, the covariant derivative of a scalar function, say $g_{\text {ret }}^{\Lambda}(\sigma)$, is

$$
\begin{aligned}
\nabla_{\alpha} g_{r e t}^{\Lambda}(\sigma) & =\sigma_{\alpha}\left(\frac{\partial \sigma}{\partial s}\right)^{-1} \frac{\partial g_{r e t}^{\Lambda}(\sigma)}{\partial s} \\
& =-\frac{\sigma_{\alpha}}{s} \frac{\partial g_{r e t}^{\Lambda}}{\partial s}+\mathcal{O}\left(s^{4}\right)
\end{aligned}
$$

\section{APPENDIX B: COEFFICIENTS AND VECTORS IN REGULATED DYNAMICS}

The $r$-dependent coefficients $\left(r=\tau-\tau_{i}\right)$ appearing in the equation for the regulated semi-classical dynamics (4.10) are

$$
\begin{aligned}
c_{(n)}(r) & =-\int_{0}^{r} d s \frac{(-s)^{n}}{n !} g_{r e t}^{\Lambda}(s) \\
& =(-1)^{n+1} \frac{2^{(n-1) / 4}}{\pi^{1 / 2} n !} \Lambda^{1-n} \gamma\left(\frac{1+n}{4}, \frac{r^{4} \Lambda^{4}}{2}\right)
\end{aligned}
$$

$$
\begin{aligned}
g_{(n)}(r) & =-\int_{0}^{r} d s \frac{(-s)^{n}}{n !} \frac{\partial}{\partial s} g_{r e t}^{\Lambda}(s) \\
& =(-1)^{n} \frac{2^{(n+6) / 4}}{\pi^{1 / 2} n !} \Lambda^{2-n} \gamma\left(1+\frac{n}{4}, \frac{r^{4} \Lambda^{4}}{2}\right)
\end{aligned}
$$

The corresponding worldline-dependent vectors are

$$
\begin{aligned}
u_{\mu}^{(0)}= & 0 \\
u_{\mu}^{(1)}= & \frac{1}{2} \bar{a}_{\mu} \\
u_{\mu}^{(2)}= & \frac{1}{3} w_{\mu}{ }^{\alpha} \frac{D \bar{a}_{\alpha}}{d \tau} \\
u_{\mu}^{(3)}= & \frac{1}{4} w_{\mu}{ }^{\alpha} \frac{D^{2} \bar{a}_{\alpha}}{d \tau^{2}}+\frac{1}{4} a_{\mu} R_{\alpha \beta} u^{\alpha} u^{\beta} \\
\vdots & -\bar{a}_{\mu} \\
v_{\mu}^{(0)}= & \frac{1}{6} w_{\mu}^{\alpha} R_{\alpha \beta} \bar{u}^{\beta} \\
v_{\mu}^{(1)}= & \frac{1}{6} w_{\mu}{ }^{\alpha} R_{\alpha \beta} \bar{a}^{\beta}-\frac{1}{6} \bar{a}_{\mu} R_{\alpha \beta} \bar{u}^{\alpha} \bar{u}^{\beta} \\
v_{\mu}^{(2)}= & +\frac{1}{9} R_{\mu \alpha \beta}{ }^{\gamma} ; \bar{u}^{\alpha} \bar{u}^{\beta}
\end{aligned}
$$

For finite and large $\Lambda$ the first-order correction to the self-force $f_{\alpha}[\bar{z}]$ is

$$
e^{2}\left(g_{(3)}(r) u_{\alpha}^{(3)}[\bar{z}]+c_{(2)}(r) v_{\alpha}^{(2)}[\bar{z}]\right)
$$

which in flat spacetime reduces to $\frac{e^{2}}{4} g_{(3)}(r) w_{\alpha}^{\beta}[\bar{z}] \frac{D^{2} \bar{a}_{\beta}}{d \tau^{2}}$.

\section{APPENDIX C: COEFFICIENTS IN LINEARIZED STOCHASTIC DYNAMICS}

The time- and mean worldline-dependent coefficients appearing in (15.7) as a result of linearizing the self-force $f_{\mu}$ (5.2) are

$$
\begin{gathered}
\kappa_{\mu \alpha}(\tau ; \bar{z}]=\frac{e^{2}}{3} g_{(2)}(r)\left(\frac{d}{d \tau}\left(\partial_{\bar{z}^{\alpha}} \Gamma_{\beta \nu}^{\gamma} \bar{u}^{\beta} \bar{u}_{\gamma}\right)+\partial_{\bar{z}^{\alpha}} \Gamma_{\beta \nu}^{\gamma} \bar{u}^{\beta} \bar{a}_{\gamma}-\bar{u}^{\beta} \Gamma_{\beta \nu}^{\gamma} \partial_{\bar{z}^{\alpha}} \Gamma_{\gamma \delta}^{\epsilon} \bar{u}^{\delta} \bar{u}_{\epsilon}\right) \\
-w_{\mu}^{\nu}[\bar{z}]\left(\frac{e^{2}}{6} c_{(1)}(r) \bar{u}^{\beta} \nabla_{\bar{z}^{\alpha}} R_{\nu \beta}+e \nabla_{\bar{z}^{\alpha}} \phi_{\nu}^{t a i l}(\bar{z})\right) \\
\gamma_{\mu \alpha}(\tau ; \bar{z}]=\frac{e^{2}}{3} g_{(2)}(r) w_{\mu}^{\nu}[\bar{z}]\left[2 \frac{d}{d \tau}\left(\Gamma_{\beta \nu}^{\gamma} \bar{u}^{(\beta)}\right) g_{\gamma) \alpha}+\partial_{\bar{z}^{\alpha}} \Gamma_{\beta \nu}^{\gamma} \bar{u}^{\beta} \bar{u}^{\gamma}+\Gamma_{\alpha \nu}^{\gamma} \bar{a}_{\gamma}-2 \bar{u}^{\beta} \Gamma_{\beta \nu}^{\gamma} \Gamma_{\gamma \delta}^{\epsilon} \bar{u}^{(\delta} g_{\epsilon) \alpha}\right] \\
-\frac{e^{2}}{6} w_{\mu}^{\nu}[\bar{z}] c_{(1)}(r) R_{\nu \alpha}-2 \bar{u}^{(\nu} g_{\mu) \alpha}\left(\frac{e^{2}}{3} g_{(2)}(r) \frac{D \bar{a}_{\nu}}{d \tau}+\frac{e^{2}}{6} c_{(1)}(r) R_{\nu \beta} \bar{u}^{\beta}+e \phi_{\nu}^{t a i l}(\bar{z})\right) \\
m_{\mu \alpha}(\tau ; \bar{z}]=\frac{e^{2}}{3} g_{(2)}(r) w_{\mu}^{\nu}[\bar{z}]\left(2 \Gamma_{\beta \nu}^{\gamma} \bar{u}^{(\beta} g_{\gamma) \alpha}+\bar{u}^{\beta} \Gamma_{\beta \nu}^{\gamma} g_{\gamma \alpha}\right)
\end{gathered}
$$




$$
r_{\mu \alpha}(\tau ; \bar{z}]=\frac{e^{2}}{3} g_{(2)}(r) w_{\mu \alpha}[\bar{z}]
$$

\section{APPENDIX D: NOISE-INDUCED DRIFT}

In this section we outline the details for obtaining the noise-induced force on the particle (5.13). We begin with the ALD-Langevin equation with noise added in by hand

$$
m_{r e n}[z] \frac{D u_{\mu}}{d \tau}=F_{\mu}^{e x t}(\tau)+f_{\mu}[z]+\eta_{\mu}^{+}[z]
$$

If we expand the worldline coordinates in powers of the coupling constant $e$

$$
z^{\mu}=z_{0}^{\mu}+z_{1}^{\mu}+z_{2}^{\mu}+\ldots
$$

where the subscript denotes the order of the expansion, then to lowest order one finds

$$
m_{r e n} \frac{D_{0} u_{0 \mu}}{d \tau}=F_{\mu}^{e x t}(\tau)
$$

where $D_{0} / d \tau$ is the covariant $\tau$-derivative defined with respect to $z_{0}^{\mu}$. If the external force vanishes then this is nothing more than the geodesic equation.

The first order correction to the trajectory can be found by solving

$$
\begin{gathered}
\frac{d u_{1 \mu}}{d \tau}-2 \Gamma_{\alpha \mu}^{\beta}\left(z_{0}\right) u_{0}^{(\alpha} u_{1 \beta)}-z_{1}^{\gamma} \partial_{z_{0}^{\gamma}} \Gamma_{\alpha \mu}^{\beta}\left(z_{0}\right) u_{0}^{\alpha} u_{0 \beta} \\
=\frac{\eta_{\mu}^{+}\left(z_{0}\right)}{m_{\text {ren }}}
\end{gathered}
$$

which has the formal solution

$$
z_{1}^{\mu}(\tau)=\int d \tau^{\prime} g_{r e t}^{\mu \nu}\left(\tau, \tau^{\prime}\right) \eta_{\nu}^{+}\left(z_{0}^{\alpha^{\prime}}\right)
$$

and $g_{r e t}^{\mu \nu}$ is the retarded Green's function for $z_{1}$.

To second order in $e$ we find that $z_{2}$ satisfies

$$
\begin{aligned}
m_{r e n}\left(\frac{d u_{2 \mu}}{d \tau}-2 \Gamma_{\alpha \mu}^{\beta}\left(z_{0}\right)\right. & \left.u_{0}^{(\alpha} u_{2 \beta)}-z_{2}^{\gamma} \partial_{z_{0}^{\gamma}} \Gamma_{\alpha \mu}^{\beta}\left(z_{0}\right) u_{0}^{\alpha} u_{0 \beta}\right) \\
= & e^{2} \frac{F_{\mu}^{e x t}}{m_{r e n}} \int_{\tau_{i}}^{\tau} d \tau^{\prime} V\left(z_{0}^{\alpha}, z_{0}^{\alpha^{\prime}}\right)+f_{\mu}\left[z_{0}\right]+z_{1}^{\nu} \nabla_{z_{0}^{\nu}} \eta_{\mu}^{+}\left(z_{0}\right) \\
& \left.\quad+m_{r e n}\left(\Gamma_{\alpha \mu}^{\beta}\left(z_{0}\right) u_{1}^{\alpha} u_{1 \beta}+2 z_{1}^{\gamma} \partial_{z_{0}^{\gamma}} \Gamma_{\alpha \mu}^{\beta}\left(z_{0}\right) u_{1}^{(\alpha} u_{0 \beta}\right)+\frac{1}{2} z_{1}^{\gamma} z_{1}^{\delta} \partial_{z_{0}^{\gamma}} \partial_{z_{0}^{\delta}} \Gamma_{\alpha \mu}^{\beta}\left(z_{0}\right) u_{0}^{\alpha} u_{0 \beta}\right)
\end{aligned}
$$

Taking the stochastic average we find that the terms after the self-force give a non-vanishing contribution to the force on the particle

$$
F_{\mu}^{d r i f t}(\tau)=\left\langle z_{1}^{\nu} \nabla_{z_{0}^{\nu}} \eta_{\mu}^{+}\left(z_{0}\right)+m_{r e n}\left(\Gamma_{\alpha \mu}^{\beta} u_{1}^{\alpha} u_{1 \beta}+2 z_{1}^{\gamma} \partial_{z_{0}^{\gamma}} \Gamma_{\alpha \mu}^{\beta} u_{1}^{(\alpha} u_{0 \beta}+\frac{1}{2} z_{1}^{\gamma} z_{1}^{\delta} \partial_{z_{0}^{\gamma}} \partial_{z_{0}^{\delta}} \Gamma_{\alpha \mu}^{\beta} u_{0}^{\alpha} u_{0 \beta}\right)\right\rangle_{\eta^{+}}
$$

Substituting in for $z_{1}$ we find that $F_{\mu}^{d r i f t}$ can be written in terms of the noise kernel associated with $\eta_{\mu}^{+}$

$$
\begin{aligned}
F_{\mu}^{d r i f t}(\tau)= & \int d \tau^{\prime} g_{\text {ret }}^{\nu \sigma^{\prime}} \nabla_{z_{0}^{\nu}}\left\langle\eta_{\mu}^{+}\left(z_{0}^{\lambda}\right) \eta_{\sigma^{\prime}}^{+}\left(z_{0}^{\lambda^{\prime}}\right)\right\rangle_{\eta^{+}} \\
+ & m_{\text {ren }} \int d \tau^{\prime} d \tau^{\prime \prime}\left\{\frac{1}{2} \partial_{z_{0}^{\gamma}} \partial_{z_{0}^{\delta}} \Gamma_{\alpha \mu}^{\beta}\left(z_{0}^{\lambda}\right) u_{0 \beta} g_{\text {ret }}^{\gamma \rho^{\prime}} g_{r e t}^{\delta \sigma^{\prime \prime}}+2 \partial_{z_{0}^{\gamma}} \Gamma_{\alpha \mu}^{\beta}\left(z_{0}^{\lambda}\right) g_{r e t}^{\gamma \rho^{\prime}} u_{0(\beta} \frac{d}{d \tau} g_{\text {ret }}^{\alpha) \sigma^{\prime \prime}}\right. \\
& \left.\quad+\Gamma_{\alpha \mu}^{\beta}\left(z_{0}^{\lambda}\right)\left(\frac{d}{d \tau} g_{r e t}^{\alpha \rho^{\prime}}\right)\left(\frac{d}{d \tau} g_{\text {ret } \beta} \sigma^{\prime \prime}\right)\right\}\left\langle\eta_{\rho^{\prime}}^{+}\left(z_{0}^{\lambda^{\prime}}\right) \eta_{\sigma^{\prime \prime}}^{+}\left(z_{0}^{\lambda^{\prime \prime}}\right)\right\rangle_{\eta^{+}}
\end{aligned}
$$

Notice that the first term in (D7) comes from linearizing the stochastic force whereas the other terms have their origins in the kinetic term $m[z] a_{\mu}$. Finally, we arrive at (5.13) by defining the kernel $F_{\mu}^{\rho \sigma}$ as the integrand appearing above

$$
F_{\mu}^{d r i f t}(\tau)=\int d \tau^{\prime} d \tau^{\prime \prime} F_{\mu}^{\rho \sigma}\left(\tau, \tau^{\prime}, \tau^{\prime \prime}\right)\left\langle\eta_{\rho}^{+}\left(z_{0}^{\lambda^{\prime}}\right) \eta_{\sigma}^{+}\left(z_{0}^{\lambda^{\prime \prime}}\right)\right\rangle_{\eta^{+}}
$$


[1] T. C. Quinn, Phys. Rev. D 62, 064029 (2000).

[2] B. S. DeWitt and R. W. Brehme, Ann. Phys. (N.Y.) 9, 220 (1960).

[3] Y. Mino, M. Sasaki and T. Tanaka, Phys. Rev. D 55, 3457 (1997).

[4] T. C. Quinn and R. M. Wald, Phys. Rev. D 56, 3381 (1997).

[5] S. Detweiler and B. F. Whiting, Phys. Rev. D 67, 024025 (2003).

[6] E. Poisson, "The Motion of Point Particles in Curved Spacetime", Living Rev. Relativity 7, (2004), 6. URL (cited on 26 March 2004): $\quad$ http://www.livingreviews.org/lrr-2004-6 gr-qc/0306052 ; S. Detweiler, "Perspective on gravitational self-force analyses" gr-qc/0501004.

[7] A. Raval, B.L. Hu and J. Anglin, Phys. Rev. D 53, 7003 (1996).

[8] B. L. Hu, Alpan Raval, "Is there emitted radiation in Unruh effect?" Invited Talk at the Capri Workshop on Quantum Aspects of Beam Physics, Oct. 2000 . Proceedings edited by Pisin Chen.(World-Scientific, Singapore, 2001) quant-ph/0012134 and references therein.

[9] S. Y. Lin and B. L. Hu, "Accelerated Detector - Quantum Field Correlations I. From Vacuum Fluctuations to Radiation Flux" [quant-ph/0505xxx]

[10] P. Johnson and B. L. Hu, quant-ph/0012137 (2000); P. R. Johnson and B. L. Hu, Phys. Rev. D 65, 065015 (2002).

[11] A. Raval, B. L. Hu and D. Koks, Phys. Rev. D 55, 4795 (1997).

[12] Alpan Raval, "Stochastic Properties of Particle Detector and Quantum Field Interactions" University of Maryland Ph.D. Dissertation Dec. 1996.

[13] P. Johnson, "Nonequilibrium Dynamics of Particle Field Interaction" University of Maryland Ph.D. Dissertation Dec. 1999.

[14] Philip R. Johnson and B. L. Hu, "Unruh Effect in a Uniformly Accelerated Charge: From quantum fluctuations to classical radiation" Foundations of Physics (2005) gr-qc/0501029 and references therein.

[15] B.L. Hu, J.P. Paz and Y. Zhang, Phys. Rev. D 45 , 2843 (1992).

[16] H. Grabert, P. Schramm and G.L. Ingold, Phys. Rep. 168, 115 (1988).

[17] L. D. Romero and J.P. Paz, Phys. Rev. A 55, 4070 (1997).

[18] E. Calzetta, A. Roura and E. Verdaguer, Physica A 319, 188 (2003), quant-ph/0011097.

[19] L. M. Burko, A. I. Harte and E. Poisson, Phys. Rev. D 65, 124006 (2002).

[20] L. Barack and A. Ori, Phys. Rev. D 61, 061502(R) (2000).

[21] L. Barack, Phys. Rev. D 62, 084027 (2000); L. Barack, Phys. Rev. D 62, 084027 (2000).

[22] L. Barack, Y. Mino, H. Nakano, A. Ori and M. Sasaki, Phys. Rev. Lett. 88, 091101 (2002).

[23] L. Barack and A. Ori, Phys. Rev. D 66, 084022 (2002); L. Barack and A. Ori, Phys. Rev. D 67, 024029 (2003).

[24] Y. Mino, H. Nakano, and M. Sasaki, Prog. Theor. Phys. 108, 1039 (2003).
[25] E. Rosenthal, Phys. Rev. D 69, 064035 (2004).

[26] F. Rohrlich, Am. J. Phys. 28, 639 (1969); E. Moniz and D. Sharp, Phys. Rev. D 15, 2850 (1977); P.W. Milonni, Phys. Lett. 82A, 225 (1981).

[27] N.V. Kampen, K. Dan. Vidensk. Selsk. Mat. Fys. Medd. 26 No. 15, 1 (1951); E. Moniz and D. Sharp, Phys. Rev. D 10, 1133 (1974); H. Levine, E. Moniz and D. Sharp, Am. J. Phys. 45, 75 (1977).

[28] W. Goldberger and I. Rothstein, hep-th/0409156].

[29] J. Koga, Phys. Rev. E 70, 046502 (2004).

[30] L. Barack and L. M. Burko, Phys. Rev. D 62084040 (2000); L. M. Burko, Phys. Rev. D 67084001 (2003); S. Detweiler, E. Messaritaki and B. F. Whiting, Phys. Rev. D 67104016 (2003).

[31] L. D. Landau and E. M. Lifshitz, The Classical Theory of Fields (Butterworth-Heinemann, Oxford, 1999).

[32] R. B. Griffiths, J. Stat. Phys. 36, 219 (1984); R. Omnés, J. Stat Phys. 53, 893 (1988); ibid. 53933 (1988); ibid. 53 957 (1988); ibid. 54, 357 (1988); Ann. Phys. (NY) 201, 354 (1990); Rev. Mod. Phys. 64, 339 (1992); The Interpretation of Quantum Mechanics (Princeton UP, Princeton, 1994); M. Gell-Mann and J. B. Hartle, in Complexity, Entropy and the Physics of Information, ed. by W. H. Zurek (Addison-Wesley, Reading, 1990); M. Gell-Mann and J. B. Hartle, Phys. Rev. D 47, 3345 (1993); J. B. Hartle, "Quantum Mechanics of Closed Systems" in Directions in General Relativity Vol. 1, eds B. L. Hu, M. P. Ryan and C. V. Vishveswara (Cambridge Univ., Cambridge, 1993); H. F. Dowker and J. J. Halliwell, Phys. Rev. D 46, 1580 (1992); J. J. Halliwell, Phys. Rev. D 48, 4785 (1993); ibid. 57, 2337 (1998); T. A. Brun, Phys. Rev. D 47, 3383 (1993); J. P. Paz and W. H. Zurek, Phys. Rev. D 482728 (1993); J. Twamley, Phys. Rev. D 48, 5730 (1993).

[33] E. Calzetta and B.L. Hu, "Decoherence of Correlation Histories" in Directions in General Relativity Vol. 2, eds B. L. Hu and T. A. Jacobson (Cambridge Univ., Cambridge, 1993) pp. 38-65.

[34] For reviews, see, e.g., B. L. Hu and E. Verdaguer, "Stochastic gravity: A primer with applications", Class. Quant. Grav. 20 (2003) R1-R42 gr-qc/0211090; "Stochastic gravity: Theory and Applications", in Living Reviews in Relativity 7 (2004) 3; article number lrr-20043 gr-qc/0307032

[35] B.L. Hu, A. Roura and E. Verdaguer, Phys. Rev. D 70, 044002 (2004).

[36] P. K. Rashevskii, Riemannian Geometry and Tensor Analysis (Nauka, 1964).

[37] In general, for systems with a nonlinear coupling to the environment or for non-Gaussian initial states of the environment the interpretation of $P_{\xi}$ as a probability distribution is not always possible. It may take on negative values in which case $P_{\xi}$ should be interpreted as a pseudoprobability distribution in a similar vein as the Wigner function [18].

[38] Recently, the authors of [28] have used the methods of effective field theory to describe the gravitational twobody problem of extended objects as an effective pointparticle theory. 